Pacific Journal of Mathematics

LIE ALGEBRAS, COALGEBRAS AND RATIONAL HOMOTOPY 


\title{
LIE ALGEBRAS, COALGEBRAS AND RATIONAL HOMOTOPY THEORY FOR NILPOTENT SPACES
}

\author{
JOSEPH NEISENDORFER
}

\begin{abstract}
This paper establishes that the homotopy category of rational differential graded commutative coalgebras is equivalent to the homotopy category of rational differential graded Lie algebras which have a nilpotent completion as homology. This generalizes a result which Quillen proved in the simply connected case. When combined with Sullivan's work on rational homotopy theory, our result shows that the homotopy category of rational differential graded Lie algebras with nilpotent finite type homology is equivalent to the rational homotopy category of nilpotent topological spaces with finite type rational homology.

Our results include the construction of minimal Lie algebra models for simply connected spaces, and we show that the rational homotopy groups of a simply connected $C W$ complex may be calculated from a free Lie algebra generated by the cells with a differential given on generators by the attaching maps.
\end{abstract}

o. Introduction. Sullivan [4] and Bousfield-Gugenheim [2] have demonstrated the equivalence of two categories: (a) the rational homotopy category of nilpotent spaces with rational homology of finite type, and (b) the homotopy category of commutative, associative, differential graded rational algebras with minimal models of finite type. Earlier, Quillen [23] had demonstrated the equivalence of the rational homotopy category of simply connected spaces with two categories (among others): (c) the homotopy category of commutative, associative, differential graded simply connected rational coalgebras, and (d) the homotopy category of differential graded connected rational Lie algebras. In this paper, we combine these two approaches and generalize the above portion of Quillen's work to nilpotent spaces with rational homology of finite type (Proposition 7.3).

Since we are developing a theory for nilpotent spaces, nilpotent Lie algebras play an important role. As a consequence of our work, we get the purely algebraic result that the homotopy category of commutative differential graded coalgebras is equivalent to the homotopy category of differential graded Lie algebras whose homology is a nilpotent completion (Definition 3.4 and Proposition 7.2).

We associate to each nilpotent space $X$ with rational homology of finite type, three types of differential graded models, a minimal algebra $M_{X}$, a minimal coalgebra $C_{X}$, and a free Lie algebra $\mathscr{L}\left(C_{X}\right)$. 
(The minimal algebra is due to Sullivan [4].) When $X$ is simply connected, we can replace $\mathscr{L}\left(C_{X}\right)$ by a minimal Lie algebra $L_{X}$. All three minimal models are unique up to isomorphism and determine the rational homotopy type of $X$ (Proposition 8.1).

John Moore (unpublished), Baues and Lemaire (Minimal models in homotopy theory, preprint), and Tim Miller [14] have given independent constructions of minimal Lie algebras.

If we forget the differential, the minimal Lie algebra model $L_{X}$ is a free Lie algebra on the desuspension of the rational homology of $X$ (Proposition 8.4). The homology of $L_{X}$, or of $\mathscr{L}\left(C_{X}\right)$ when $X$ is not required to be simply connected, is $\pi_{n+1}(X) \otimes Q$ for $n \geqq 1$ and $H_{0} \mathscr{L}\left(C_{X}\right)$ is the Lie algebra associated to the Malcev completion of the fundamental group $\pi_{1}(X)$. (Propositions 8.2 and 8.3.) These results are dual, in the sense of Hilton and Eckmann, to Sullivan's description of minimal algebras.

As a consequence of a Hirsch lemma (Proposition 8.11) for Lie algebras, we get a $C W$ theory for rational homotopy (Proposition 8.12). More precisely, there is a differential on the free Lie algebra $F$ generated by the desuspended cells of a simply connected $C W$ complex $X$ such that $H F=\pi(\Omega X) \otimes Q$.

After the basic definitions and lemmas in $\S 1, \S 2$ shows that there is a Lie algebra structure on the homology of the primitives of a symmetric coalgebra and a coalgebra structure on the homology of the generators of a free Lie algebra. When applied to the coalgebra and Lie algebra models, these structures correspond to Whitehead products in rational homotopy and comultiplication in rational homology, respectively.

Section 3 studies extension sequences of coalgebras. The main result is that a symmetric coalgebra is constructible by nonprimitive elementary extensions (Definition 3.9), modulo tensoring with an acyclic symmetric coalgebra, if and only if the Lie algebra structure defined in $\S 2$ is a nilpotent completion (Proposition 3.12). This is the algebraic analogue of representing a nilpotent space as an inverse limit of principal fibrations. What we do here was motivated by [4] and is a generalization of results that John Moore [18] proved for simply connected coalgebras.

Section 4 discusses certain adjoint functions $\mathscr{L}$ and $\mathscr{C}$ between differential graded coalgebras and Lie algebras. The main result is that the adjunction maps are homology isomorphisms with no restriction to simply connected coalgebras (Proposition 4.1). This result has been published [Moore, 17] but without proof, so we give one.

In $\S 5$, we introduce Quillen's closed model category concepts and discuss fibrant, cofibrant, and minimal models (Definitions 5.4 and 5.5), ending in proofs of existence, uniqueness, and a complete 
algebraic characterization (Propositions 5.6 to 5.9).

Section 6 defines homotopy groups for algebras, coalgebras, and Lie algebras. This is essentially a combination of the structures defined in $\S 2$ with the concepts introduced in $\S 5$. Our definition of the homotopy Lie algebra of a coalgebra differs from that preferred by John Moore [19]. In particular, our homotopy Lie algebra is always a nilpotent completion (Proposition 6.2) and is invariant under homology isomorphism of coalgebras.

Sections 7 and 8 contain the results mentioned at the beginning of this introduction relating algebras, coalgebras, and Lie algebras to rational homotopy theory. These are the main results of the paper and the reader who seeks motivation should read those sections first.

The main point of this paper is to establish the equivalence between differential graded rational Lie algebras and rational homotopy theory as expounded by Sullivan. In other papers, [15] and [20], these results are applied to the study of Sullivan's formal spaces, i.e., those whose rational homotopy type is a formal consequence of their rational cohomology rings. Minimal Lie algebra models can be used to show that certain spaces are formal, e.g., every $n$ connected compact manifold of dimension $\leqq 3 n+1, n \geqq 1$ [15]. Galois cohomology applies to minimal Lie algebras to prove: Any simply connected space which is formal over an extension field of the rationals is necessarily rationally formal [15].

Most importantly, minimal Lie algebras can be used to give complete calculations of the rational homotopy groups of some quite complicated spaces. For example, in [20] I calculate the rational homotopy Lie algebra of all projective algebraic varieties which are complete intersections of complex dimension $\geqq 2$.

In Quillen's original approach to rational homotopy theory for simply connected spaces, there were no finite type restrictions on homology. We suspect that Quillen's approach may be generalized to nilpotent spaces with no finite type restrictions. But this removal of finite type restrictions would be achieved at the cost of the connection between Quillen's work and Sullivan's work. Many applications would be lost and the treatment would be significantly longer. So we chose to keep the connection.

Besides the obvious debts to authors already mentioned in this introduction, I owe much to Alison Beall, Tim Miller, John Moore, Jim Stasheff, and Larry Taylor for stimulating and encouraging conversations.

1. Algebras, coalgebras, and Lie algebras. This section presents basic definitions and lemmas concerning differential graded algebras, coalgebras, and Lie algebras. Good references are [16], [10], and [23]. 
Let $k$ be a field of characteristic zero.

$D G A$ is the category of differential graded, augmented, commutative, associative algebras defined over $k$ which are concentrated in nonnegative degrees and have a cohomology differential (degree +1 ). We restrict $D G A$ to algebras $A$ which are homologically connected, $H^{\circ}(A)=k$. We call the objects in this category algebras and shall suppress the augmentations $A \rightarrow k$ in our notation.

The category $D G A$ has coproducts $A \otimes B$ and products $A \vee B=$ the equalizer of the maps $A \oplus B \rightarrow A \rightarrow k$ and $A \oplus B \rightarrow B \rightarrow k$.

Recall that $A=$ the kernel of $A \rightarrow k$ and $Q(A)(=\bar{A} / \bar{A} \cdot \bar{A})$ is the module of indecomposables.

$D G C$ is the category of differential graded, connected, commutative, associative coalgebras defined over $k$ which are concentrated in nonnegative degrees and have a homology differential (degree -1 ). We call the objects in this category coalgebras and note that connected coalgebras $C$ have a unique augmentation $k \rightarrow C$.

The category $D G C$ has products $C \otimes D$ and coproducts $C \vee D=$ the coequalizer of the maps $k \rightarrow C \rightarrow C \oplus D$ and $k \rightarrow D \rightarrow C \oplus D$.

Recall that $\bar{C}=$ the cokernel of $k \rightarrow D$ and $P(C)$ (=the kernel of $\bar{C} \rightarrow \bar{C} \otimes \bar{C}$ ) is the module of primitives.

$D G L A$ is the category of differential graded Lie algebras defined over $k$ which are concentrated in nonnegative degrees and have a homology differential. We will call objects in this category Lie algebras.

For the convenience of the reader, we recall the identities for a graded Lie bracket [ ]: $L \otimes L \rightarrow L$ over a field of characteristic zero [11] [23].

(antisymmetry) $[x, y]=-(-1)^{\operatorname{deg} x \operatorname{deg} y}[y, x]$

(Jacobi identity) $[x,[y, z]]=[[x, y], z]+(-1)^{\operatorname{deg} x \operatorname{deg} y}[y,[x, z]]$

Notice that, whenever $A$ is an associative algebra, defining $[x, y]=x y-(-1)^{\operatorname{deg} x \operatorname{deg} y} y x$ makes $A$ into a Lie algebra.

The reader may dualize these identities to obtain the definition of a Lie coalgebra.

The category $D G L A$ has products $L \times L^{\prime}$ and coproducts $L \vee L^{\prime}=$ free product of $L$ and $L^{\prime}$.

Quillen [23] has shown:

Proposition 1.1. Lie algebras $L$ have universal enveloping Hopf algebras $U(L)$ with natural isomorphisms $L \cong P U(L)$ and $H L \cong$ $P H U(L)$.

Definition 1.2. For a Lie algebra $L$, we define $Q(L)=L /[L, L]=$ the abelianization of $L$. 
Let $V$ be a graded vector space with a differential. (The case of no differential is included, as always, by imposing the zero differential.) In $D G A, D G C$, or $D G L A$ we shall use various objects $S[V], S^{\prime}[V]$, or $F[V]$ which we shall now describe.

$T[V]=\bigoplus V^{\otimes n}$ is the tensor algebra with multiplication $V^{\otimes n} \otimes$ $V^{\otimes m}=V^{\otimes(n+m)}, \quad\left(x_{1} \otimes \cdots \otimes x_{n}\right)\left(x_{n+1} \otimes \cdots \otimes x_{n+m}\right)=x_{1} \otimes \cdots \otimes x_{n+m}$. This $\overrightarrow{\text { is }}$ not a commutative algebra. The quotient of $T[V]$ by the ideal generated by all $x \otimes y-(-1)^{\operatorname{deg} x \operatorname{deg} y} y \otimes x$, where $x$ and $y$ are in $V$, is a commutative algebra, denoted $S[V]$.

The symmetric group acts on each $V^{\otimes n}$ by permuting the tensors with the usual sign and the projection $T[V] \rightarrow S[V]$ has a canonical splitting $\sigma: S[V] \rightarrow T[V]$ onto the set of invariant elements, e.g., $\sigma(x y)=1 / 2\left(x \otimes y+(-1)^{\operatorname{deg} x \operatorname{deg} y} y \otimes x\right)$. The map $\sigma: S[V] \rightarrow T[V]$ is not an algebra map.

$S[V]$ is called the free commutative or symmetric algebra generated by $V$ and is characterized up to isomorphism by the universal property: Given any linear map $f: V \rightarrow B$, where $B$ is a (commutative) algebra, there exists a unique algebra map $S[V] \rightarrow B$ such that $V \rightarrow S[V] \rightarrow B$ equals $f$.

$T^{\prime}[V]=\bigoplus V^{\otimes n}$ is the tensor coalgebra with comultiplication $V^{\otimes r} \rightarrow \bigoplus_{m+n=r}\left(V^{\otimes n} \otimes V^{\otimes n}\right), \quad x_{1} \otimes \cdots \otimes x_{r} \rightarrow \Sigma\left(x_{1} \otimes \cdots \otimes x_{m}\right) \otimes$ $\left(x_{m+1} \otimes \cdots \otimes x_{r}\right)$.

This is not a commutative coalgebra. The subspace $S^{\prime}[V]$ of invariant elements is a commutative subcoalgebra of $T^{\prime}[V]$, and there is a canonical retraction $\sigma^{\prime}: T^{\prime}[V] \rightarrow S^{\prime}[V]$, e.g., $\sigma^{\prime}(x \otimes y)=$ $1 / 2\left(x \otimes y+(-1)^{\operatorname{deg} x \operatorname{deg} y} y \otimes x\right)$. The map $\sigma^{\prime}$ is not a coalgebra map.

$S^{\prime}[V]$ is called the injective commutative or symmetric coalgebra cogenerated by $V$ and is characterized up to isomorphism by the universal property: Given any linear map $g: D \rightarrow V$, where $D$ is a (commutative) coalgebra, there exists a unique coalgebra map $D \rightarrow$ $S^{\prime}[V]$ such that $D \rightarrow S^{\prime}[V] \rightarrow V$ equals $g$.

Remarks. As $S[V]$ was characterized by every map defined on $V$ extending uniquely, so $S^{\prime}[V]$ is characterized by every map into $V$ lifting uniquely.

$F[V]$ denotes the free Lie algebra generated by $V$ and is characterized up to isomorphism by the universal property: Given any linear map $f: V \rightarrow L$, where $L$ is a Lie algebra, there exists a unique Lie algebra map $F[V] \rightarrow L$ such that $V \rightarrow F[V] \rightarrow L$ equals $f$.

$F[V]$ may also be described as follows: [23] Make the tensor algebra $T[V]$ into a Hopf algebra by defining the comultiplication on $x$ in $V$ by $x \rightarrow x \otimes 1+1 \otimes x$. Then $F[V]=P T[V], T[V]=$ $U F[V]$. 
LEMMA 1.3. If $A, C$, or $L$ is a connected symmetric algebra, symmetric coalgebra, or free Lie algebra, respectively, then so is any retract.

This lemma is a simple consequence of the definitions by universal mapping properties.

We close this section with a collection of technical lemmas for (connected) coalgebras. These lemmas are the duals of well known results for algebras and the proofs will be left as exercises.

If $D$ is a coalgebra, we may pick a splitting $D \rightarrow P D$ for the inclusion $P D \rightarrow D$.

LemMa 1.4. Two coalgebra maps $C \rightarrow D$ are equal if and only if the two compositions $C \rightarrow D \rightarrow P D$ are equal.

LEMMA 1.5. Given a coalgebra map $C \rightarrow D$ and a surjective coalgebra map $C \rightarrow C^{\prime}$, then $C \rightarrow D$ factors into $C \rightarrow C^{\prime} \rightarrow D$ if and only if $C \rightarrow D^{\prime} \rightarrow P D$ factors into $C \rightarrow C^{\prime} \rightarrow P D$.

If $C$ is a coalgebra, a coderivation on $C$ is a degree -1 linear map $d: C \rightarrow C$ such that the following diagrams commute.
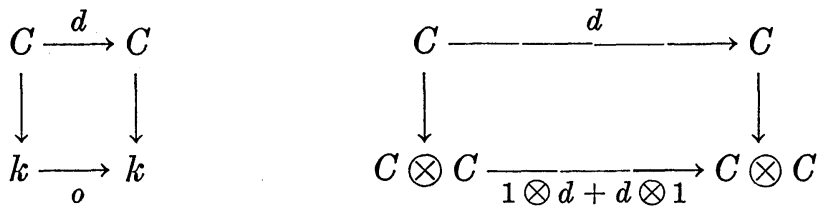

Note that a $D G$ coalgebra is just a coalgebra with a coderivation $d$ such that $d^{2}=0$.

LEMMA 1.6. Two coderivations on a coalgebra $C$ are equal if and only if the two compositions $C \rightarrow C \rightarrow P C$ are equal.

LEMMA 1.7. Given a coderivation $d$ on a coalgebra $C$ and $a$ surjective coalgebra map $f: C \rightarrow C^{\prime}$, then there exists a coderivation $d^{\prime}$ on $C^{\prime}$ such that $d^{\prime} f=f d$ if and only if there exists a linear map $e: C^{\prime} \rightarrow P C^{\prime}$ such that ef $=\pi f d$ where $\pi: C^{\prime} \rightarrow P C^{\prime}$ is a splitting.

LeMMA 1.8. A coderivation $d$ on $C$ satisfies $d^{2}=0$ if and only if $\pi d^{2}=0$ where $\pi: C \rightarrow P C$ is a splitting.

Lemma 1.9. Given a degree -1 linear map $\bar{d}: S^{\prime}[V] \rightarrow V$, there exists a unique coderivation $d$ on $S^{\prime}[V]$ such that $\pi d=\bar{d}$. 
Lemma 1.10. (a) If $S^{\prime}[V]$ is a symmetric coalgebra with $H V=$ 0 , then $H S^{\prime}[V]=k$.

(b) If $S^{\prime}[V] \rightarrow S^{\prime}[W]$ is a map of symmetric coalgebras with $H V \rightarrow H W$ an isomorphism, then $H S^{\prime}[V] \rightarrow H S^{\prime}[W]$ is an isomorphism.

Finally, we remark that John Moore (unpublished) has pointed out that the category $D G C$ is closed under inverse limits but these inverse limits are not constructed in the obvious way. Hence, it is worth recording the following:

LEMMA 1.11. (a) If $V_{\alpha}$ is an inverse system of graded vector spaces, then $\lim S^{\prime}\left[V_{\alpha}\right]=S^{\prime}\left[\lim V_{\alpha}\right]$.

(b) In $\overleftarrow{D G} C$ an inverse limit of injective maps is the "intersection."

2. Lie bracket and coproduct structures. Let $C$ be a coalgebra (algebra, or Lie algebra). We shall denote by $C^{\#}$ the coalgebra (algebra, or Lie algebra) which is identical to $C$ but has zero differential.

Suppose $C$ is a coalgebra with $C^{\#}=$ a symmetric coalgebra $S^{\prime}[V]$. Consider the primitive filtration on $C$ [16], i.e., $F^{\circ} C=k, F^{1}(C)=$ $k \oplus P(C), \cdots, F^{n}(C)=k \oplus \operatorname{ker}\left(\bar{\triangle}: \bar{C} \rightarrow \bar{C}^{\otimes(n+1)}\right), \cdots$. This gives rise to a homology spectral sequence of coalgebras with $E^{\circ}(C)=C^{\sharp}=$ $S^{\prime}[P C], E^{1}(C)=S^{\prime}[H P C], E_{1, n}^{1}=H_{n+1} P C$.

$d^{1}: E_{2, n}^{1} \rightarrow E_{1, n}^{1}$ composes with $\sigma^{\prime}: H P C \otimes H P C \rightarrow S^{\prime}[H P C]$ to give a degree -1 map \langle\rangle$: H P C \otimes H P C \rightarrow H P C$.

Letting $s^{-1}$ denote desuspension and following the standard sign convention that $f \otimes g(x \otimes y)=(-1)^{\operatorname{deg} x \operatorname{deg} g} f x \otimes g y$, we define [ ]: $s^{-1} H P C \otimes s^{-1} H P C \rightarrow s^{-1} H P C$ by the commutative diagram:

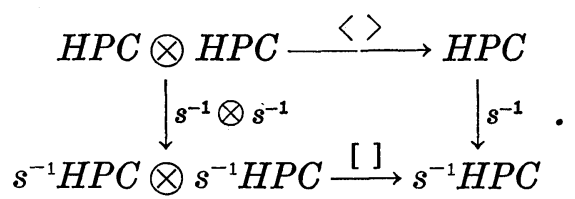

Proposition 2.1. With the above bracket, $s^{-1} H P C$ is a Lie algebra.

Proof. Since $\sigma^{\prime}$ is symmetric, \langle\rangle is also, i.e., $\langle x, y\rangle=$ $(-1)^{\operatorname{deg} x \operatorname{deg} y}\langle y, x\rangle$. Since $\left[s^{-1} x, s^{-1} y\right]=(-1)^{\operatorname{deg} x} s^{-1}\langle x, y\rangle$, if follows that [ ] is antisymmetric.

Since $\left(d^{1} \otimes 1+1 \otimes d^{1}\right)^{2} \bar{\Delta}=0$, where $\bar{\Delta}$ is the comultiplication, an elementary computation shows that: 


$$
\langle\langle x, y\rangle, z\rangle+\varepsilon\langle\langle y, z\rangle, x\rangle+\delta\langle\langle z, x\rangle, y\rangle=0
$$

where $\varepsilon=(-1)^{\operatorname{deg} x(\operatorname{deg} y+\operatorname{deg} z)}, \delta=(-1)^{\operatorname{deg} z(\operatorname{deg} x+\operatorname{deg} y)}$.

The Jacobi identity for [ ] follows.

REMARK. The binary operation \langle\rangle$: H P C \otimes H P C \rightarrow H P C$ satisfies the same identities as the Whitehead product $\pi_{n}(X) \otimes \pi_{m}(X) \rightarrow$ $\pi_{n+m-1}(X)$ in homotopy theory [30]. HPC is a "Whitehead algebra" in the sense of [21].

Dually, let $A$ be an algebra with $A^{\sharp}=$ a symmetric algebra $S[V]$. We may filter $A$ by the powers of its augmentation ideal, $F_{0}(A)_{n}=A, F_{1}(A)=\bar{A}, \cdots, F_{n}(A)=(\bar{A})^{n}, \cdots$. This gives rise to a cohomology spectral sequence of algebras with $E_{0}(A)=A^{\sharp}=S[Q A]$, $E_{1}(A)=S[H Q A], E_{1}^{1, n}=H^{n+1} Q A$.

$d_{1}: E_{1, n}^{1} \rightarrow E_{1}^{2, n}$ composes with $\sigma: S[H Q A] \rightarrow H Q A \otimes H Q A$ to give a degree +1 map \langle\rangle$^{*}: H Q A \rightarrow H Q A \otimes H Q A$. Define [ ]*: $s^{-1} H Q A \rightarrow$ $s^{-1} H Q A \otimes s^{-1} H Q A$ by the commutative diagram.

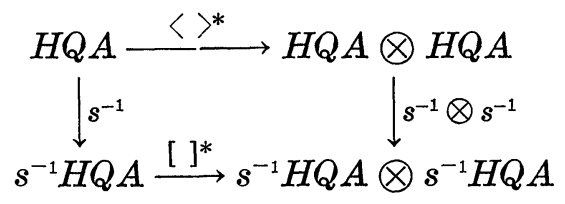

An argument similar to 2.1 shows [see 21]:

Proposition 2.2. $s^{-1} H Q A$ is a Lie coalgebra.

REMARK. If $A$ is a finite type, then $\left(s^{-1} H Q A\right)^{*}=s^{-1} H P A^{*}$ as Lie algebras.

REMARK. If the induced differential on $Q(A)$ is zero, i.e., if $d(\bar{A}) \subset \bar{A} \cdot \bar{A}$, then $Q A=H Q A$ and the Lie cobracket $s^{-1} Q A \rightarrow s^{-1} Q A \otimes$ $s^{-1} Q A$ can be read off from the quadratic term of the differential on generators [see 4 or 26].

Finally, let $L$ be a Lie algebra with $L^{\sharp}=$ a free Lie algebra $F[V]$. We may filter $L$ by the descending central series, $F^{0}(L)=L$, $F^{-1}(L)=[L, L], \cdots, F^{-n}(L)=\left[L, F^{-n+1}(L)\right], \cdots$. This gives rise to a homology spectral sequence of Lie algebras with $E^{0}(L)=L^{\sharp}=F[V]$, $E^{1}(L)=F[H V]=F[H Q L], E_{-1, n}^{1}=H_{n-1} Q L$.

$d_{1}: E_{-1, n}^{1} \rightarrow E_{-2, n}^{1}$ may be composed with $F[H Q L] \rightarrow T[H Q L] \rightarrow$ $H Q L \otimes H Q L$ to give a degree -1 map $H Q L \rightarrow H Q L \otimes H Q L$. The commutative diagram below defines a degree 0 map $\bar{\Delta}: s H Q L \rightarrow$ $s H Q L \otimes s H Q L$, where $s$ denotes suspension. 


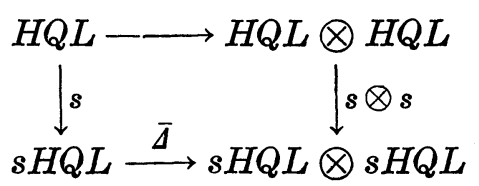

We define $\Delta$ on $k \oplus(s H Q L)$ by $\Delta(1)=1 \otimes 1, \Delta(s x)=s x \otimes 1+$ $1 \otimes s x+\bar{\Delta}(s x)$.

An argument similar to 2.1 shows:

Proposition 3.3. $k \oplus(s H Q L)$ is a (commutative) coalgebra.

A special case of 2.3 admits a nice description. Let $L$ be a Lie algebra with $L^{\#}=$ a free lie algebra $F[V]$ and suppose that $d L$ is contained in $[L, L]$. Such Lie algebras are called minimal. (See 5.5 and 5.7c.) If $x_{\alpha}$ is a basis for $V$ with $d x_{\alpha}=\Sigma c_{\beta \alpha}^{\alpha}\left[x_{\beta}, x_{\alpha}\right]+$ higher degree terms, then $\bar{\Delta}\left(s x_{\alpha}\right)=\Sigma(-1)^{\operatorname{deg} x_{\beta}} c_{\beta \alpha}^{\alpha}\left(s x_{\beta} \otimes s x_{\alpha}+(-1)^{\operatorname{deg} x_{\alpha} \operatorname{deg} x_{\beta} s x_{\alpha} \otimes}\right.$ $\left.s x_{\beta}\right)$. In other words, when $L^{\sharp}$ is free and the induced differential on $Q(L)$ is zero, then $\Delta$ can be read off from the quadratic term of the differential on $L$. Combined with $4.2 \mathrm{~b}$, this gives a complete computation of the Lie algebra homology coalgebra of a minimal Lie algebra.

3. Nilpotence. Given a graded Lie algebra $L$, recall the descending central series $F^{0}=L, F^{-1}=[L, L], \cdots$ with $F^{0} / F^{-1}=Q(L)$.

We introduce another filtration $G^{0}=L, G^{-1}=\left[L_{0}, L\right], G^{-2}=$ $\left[L_{0}, G^{-1}\right], \cdots$ and set $\widetilde{Q}(L)=G^{0} / G^{-1}$.

Definition 3.1. A graded Lie algebra $L$ is nilpotent if either of the following equivalent conditions hold:

(a) the descending central series terminates at 0 in each degree

(b) the filtration $G^{0}, G^{-1}, G^{-2}, \cdots$ terminates at 0 in each degree. Obviously, a connected Lie algebra, $L_{0}=0$, is nilpotent.

Recall the well known [16]:

LEMMA 3.2. If $f: C \rightarrow D$ is a map between coalgebras with $C$ connected, then $f$ is monic if and only if $P(f): P C \rightarrow P D$ is monic. If $f: A \rightarrow B$ is a map between algebras with $B$ connected, then $f$ is epic if and only if $Q(f): Q A \rightarrow Q B$ is epic.

For Lie algebras, we have:

LEMMA 3.3. If $f: L \rightarrow L^{\prime}$ is a map between Lie algebras with $L^{\prime}$ nilpotent, then $f$ is epic if and only if either of the following conditions hold: 
(a) $Q(f): Q(L) \rightarrow Q\left(L^{\prime}\right)$ is epic.

(b) $\widetilde{Q}(f): \widetilde{Q}(L) \rightarrow \widetilde{Q}\left(L^{\prime}\right)$ is epic.

The proof of 3.3 is a simple exercise in the use of the five lemmas.

Definition 3.4. A Lie algebra is a nilpotent completion if it is isomorphic to some $\lim L / G^{-n}$ where $L$ is a Lie algebra.

Clearly a nilpotent Lie algebra is a nilpotent completion.

We now relate nilpotence to extension sequences of coalgebras. We choose a quite restricted definition (compare [18]).

DEFINITION 3.5. An extension sequence of coalgebras is a sequence of coalgebras and maps between them $C^{\prime} \rightarrow C \rightarrow C^{\prime \prime}$ which is split when we forget the differential.

(A splitting is a coalgebra map $C \rightarrow C^{\prime}$ such that $C^{\prime} \rightarrow C \rightarrow C^{\prime}$ is the identity and such that it defines an isomorphism $C \rightarrow C^{\prime} \otimes C^{\prime \prime}$ of coalgebras.)

An extension sequence is called trivial if there is a splitting $C \rightarrow C^{\prime}$ which is a map of differential coalgebras.

$C^{\prime} \rightarrow C$ is called the fiber and $C \rightarrow C^{\prime \prime}$ the projection of the extension sequence.

Given a vector space $V_{n}$ concentrated in dimension $n \geqq 1$, define $W_{n}$ as follows:

If $n \geqq 2$, set $W_{n}=V \oplus s^{-1} V_{n}$ with $d\left(s^{-1} V_{n}\right)=0$ and $d: V_{n} \rightarrow$ $s^{-1} V_{n}$ an isomorphism.

If $n=1$, set $W_{n}=0$. (In this section, we will not use this case but it plays a role in $\S 5$.)

Let $E^{\prime}\left[V_{n}\right]=S^{\prime}\left[W_{n}\right]$ and for $n \geqq 2$, consider the extension sequence $S^{\prime}\left[s^{-1} V_{n}\right] \rightarrow E^{\prime}\left[V_{n}\right] \rightarrow S^{\prime}\left[V_{n}\right]$. If $C^{\prime \prime}$ is a coalgebra and $f:$ $C^{\prime \prime} \rightarrow V_{n}$ is a (chain) map, then $f$ defines a unique map $C^{\prime \prime} \rightarrow S^{\prime}\left[V_{n}\right]$ by the universal property of symmetric coalgebras and we may form the pullback diagram:

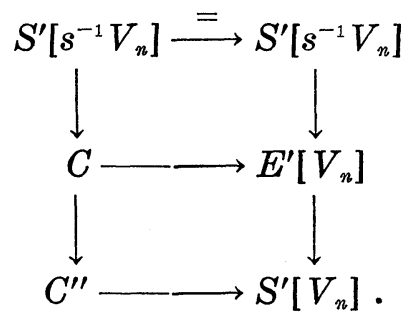

Lemma 3.6. The left hand side is an extension sequence.

Proof. The pullback is the cotensor product [16], hence $C=$ $C^{\prime \prime} \square_{S^{\prime}\left[V_{n}\right]} E^{\prime}\left[V_{n}\right]=C^{\prime \prime} \otimes S^{\prime}\left[s^{-1} V_{n}\right]$ as nondifferential coalgebras. 
Definition 3.7. An extension sequence of the type in 3.6 is called an elementary extension of dimension $n-1$. An elementary extension is called nonprimitive if the map $P C^{\prime \prime} \rightarrow C^{\prime \prime} \rightarrow V_{n}$ is zero.

The reason for introducing nonprimitive elementary extensions is contained in the next proposition.

Proposition 3.8. Let $S^{\prime}\left[s^{-1} V_{n}\right] \rightarrow C \rightarrow C^{\prime \prime}$ be an extension sequence of coalgebras with $\left(C^{\prime \prime}\right)^{\#}=a$ symmetric coalgebra. This sequence is a nonprimitive elementary extension if and only if the following sequence is short exact and a central extension of Lie algebras:

$$
0 \longrightarrow s^{-2} V_{n} \longrightarrow s^{-1} H P C \longrightarrow s^{-1} H P C^{\prime \prime} \longrightarrow 0 \text {. }
$$

Proof. First suppose that the sequence is a nonprimitive elementary extension. Recall that the cotensor product $C=C^{\prime \prime} \square_{S^{\prime}\left[V_{n}\right]} E^{\prime}\left[V_{n}\right]$ is the kernel of $\Delta \otimes 1-1 \otimes \Delta: C^{\prime \prime} \otimes E^{\prime}\left[V_{n}\right] \rightarrow C^{\prime \prime} \otimes S^{\prime}\left[V_{n}\right] \otimes E^{\prime}\left[V_{n}\right]$.

It follows from our nonprimitive hypothesis that $s^{-1} V_{n} \oplus P C^{\prime \prime} \oplus$ $\left(P C^{\prime \prime} \otimes s^{-1} V_{n}\right)$ is a differential submodule of $C$. From this it follows that the sequence of Lie algebras is short exact and a central extension.

Now suppose that the Lie algebras are short exact and a central extension. Short exactness implies that $0 \rightarrow s^{-1} V_{n} \rightarrow P C \rightarrow P C^{\prime \prime} \rightarrow 0$ is split exact as chain complexes. Let $P C \rightarrow s^{-1} V_{n}$ be a splitting. Consider the composition $C \rightarrow P C \rightarrow s^{-1} V_{n}$ where $C \rightarrow P C$ splits the inclusion $P C \rightarrow C$. (This is not a differential splitting.)

The commutative diagram

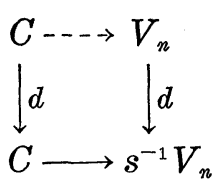

defines a chain map $C \rightarrow W_{n}$ and hence a map of differential coalgebras $C \rightarrow E^{\prime}\left[V_{n}\right]=S^{\prime}\left[W_{n}\right]$.

The composition $C \rightarrow W_{n} \rightarrow V_{n}$ factors as $C \rightarrow C^{\prime \prime} \rightarrow V_{n}$ by 1.5. The only possible obstruction is the composition $C_{1} \otimes s^{-1} V_{n} \rightarrow C \stackrel{d}{\rightarrow}$ $C \rightarrow P C \rightarrow s^{-1} V_{n}$ which is zero by the central extension hypothesis.

$C^{\prime \prime} \rightarrow V_{n}$ defines a map of differential coalgebras $C^{\prime \prime} \rightarrow S^{\prime}\left[V_{n}\right]$ and the following diagram commutes by 1.4 :

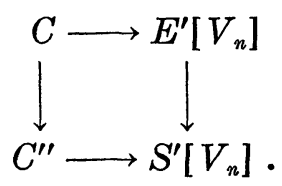


The horizontal maps are clearly isomorphisms, hence $S^{\prime}\left[s^{-1} V_{n}\right] \rightarrow$ $C \rightarrow C^{\prime \prime}$ is an elementary extension. That it is nonprimitive, i.e., that $P C^{\prime \prime} \rightarrow C^{\prime \prime} \rightarrow V_{n}$ is zero, follows from short exactness.

Definition 3.9. Let $D$ and $C$ be coalgebras. $D$ is called an inverse limit of nonprimitive elementary extensions starting from $C$ if there is an inverse system $C_{0} \leftarrow C_{1} \leftarrow C_{2} \leftarrow \ldots$ with each $C_{n} \rightarrow C_{n-1}$ the projection of a nonprimitive elementary extension, $C=C_{0}$, and $D=\lim C_{n}$.

More generally, $D$ is constructible by nonprimitive elementary extensions starting from $C$ if there is an inverse system $D_{0} \leftarrow D_{1} \leftarrow$ $D_{2} \leftarrow \cdots$ with $C=D_{0}, D=\lim _{\leftarrow} D_{n}$, and each $D_{n}$ an inverse limit of nonprimitive elementary extensions of dimension $n$ starting from $D_{n-1}$.

Definition 3.10. A coalgebra $C$ with $C^{\#}=$ a symmetric coalgebra is called a reduced symmetric coalgebra if $d=0$ on $P C$.

The next proposition is the main result of this section.

Proposition 3.11. A reduced symmetric coalgebra $C$ is constructible by nonprimitive elementary extensions starting from the ground field $k$ if and only if the Lie algebra $s^{-1} H P C=s^{-1} P C$ is a nilpotent completion.

Proof. If $C$ is constructible by nonprimitive elementary extensions starting from $k$, then it follows from 1.11a and 3.8 that $C$ is a reduced symmetric coalgebra with $s^{-1} P C$ a nilpotent completion.

Conversely, let $C$ be a reduced symmetric coalgebra with $s^{-1} P C$ a nilpotent completion. Let $C^{\sharp}=S^{\prime}[V]$ and denote by $V^{(n)}$ the graded vector space which is $V$ truncated above dimension $n$. Use 1.7 to construct an inverse system $D_{0} \leftarrow D_{1} \leftarrow D_{2} \leftarrow \cdots$ with $D_{n}^{\#}=$ $S^{\prime}\left[V^{(n)}\right]$ and $C=\lim _{\leftarrow} D_{n}$.

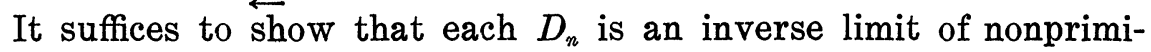
tive elementary extensions starting from $D_{n-1}$. Use 1.7 to construct an inverse system $C_{0} \leftarrow C_{1} \leftarrow C_{2} \leftarrow \cdots$ with $\left(C_{m}\right)^{\sharp}=S^{\prime}\left[X_{m}\right], X_{m}=$ $V^{(n-1)} \oplus\left(L_{n} / G^{-m} \cap L_{n}\right)$, where $G^{-m}$ is the filtration introduced at the beginning of this section and $V=\lim L / G^{-m} . \quad C_{0}=D_{n-1}$ and $D_{n}=$ $\lim C_{m}$ by 1.11a. Proposition 3.8 implies that each $C_{m} \rightarrow C_{m-1}$ is the

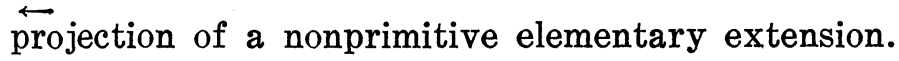

REMARK. A coalgebra constructible by nonprimitive elementary extensions starting from the ground field is automatically a reduced symmetric coalgebra.

Proposition 3.11 may be improved to: 
Proposition 3.12. Let $C$ be a coalgebra with $C^{\#}=a$ symmetric coalgebra. Then the following are equivalent.

(a) $s^{-1} H P C$ is a nilpotent completion,

(b) $C=D \otimes E$ where $D$ is a reduced symmetric coalgebra with $s^{-1} P D$ a nilpotent completion and $D=S^{\prime}[V]$ with $H V=0$.

Proof. That (b) implies (a) is trivial.

Let $s^{-1} H P C$ be a nilpotent completion and set $C^{\#}=S^{\prime}[W]$. Then $W=P C=H P C \oplus V$, where $V$ is acyclic. Since $V$ is acyclic, it is injective in the category of chain complexes [5]. Hence, the inclusion $V \rightarrow C$ has a differential splitting $C \rightarrow V$.

The map $C \rightarrow V$ defines a coalgebra map $C \rightarrow S^{\prime}[V]$. Let $D=$ $C \square_{S^{\prime}[V]} k=$ the kernel of this map $C \rightarrow S^{\prime}[V]$. Then $D^{\#}=S^{\prime}[H P C]$ and $H D \rightarrow H C$ is an isomorphism by 1.10 .

It will follow from 5.8 that $D$ is fibrant (see 5.4) and hence a homology isomorphism $D \rightarrow C$ can be split (5.11). It follows that there is an isomorphism $C \rightarrow D \otimes E$ with $E=S^{\prime}[V]$.

We leave to the reader the obvious dualization of 3.5 to 3.12 to algebras. The algebra analogue of $E^{\prime}\left[V_{n}\right]$ is denoted $E\left[V_{n}\right]$.

In particular, the dual of a nonprimitive elementary extension is just Sullivan's [4] notion of an elementary extension of algebras with decomposable differential. Just as the minimal algebras defined by Sullivan are constructible from the ground field by elementary extensions with decomposable differentials, so the minimal coalgebras (= reduced symmetric coalgebras $C$ with $s^{-1} H P C$ a nilpotent completion) to be defined in $\S 5$ are constructible by nonprimitive elementary extensions starting from the ground field. (See Proposition 5.8b.)

4. Adjoint equivalences. Quillen [23] has defined adjoint functors

$$
D G C \underset{\mathscr{C}}{\stackrel{\mathscr{L}}{\rightleftarrows}} D G L A
$$

and shown that the adjunction maps $C \rightarrow \mathscr{C} \mathscr{L}(C)$ and $\mathscr{L} \mathscr{C}(L) \rightarrow L$ are homology isomorphisms when $C$ is a simply connected coalgebra, $C_{1}=0$, and $L$ is a connected Lie algebra, $L_{0}=0$. In this section we show that these assumptions are not necessary.

$\mathscr{L}$ may be described as follows: As a Lie algebra, $\mathscr{L}(C)=$ $F\left[s^{-1} \bar{C}\right]=$ the free Lie algebra on the desuspension of $\bar{C}=\operatorname{ker}(C \rightarrow k)$. The differential on $\mathscr{L}(C)$ splits as a sum of two differentials $d=$ $d_{I}+d_{\Delta}$, where on generators $s^{-1} c, c \in \bar{C}$,

$$
\begin{aligned}
& d_{I} s^{-1} c=-s^{-1} d c \\
& d_{\Delta} s^{-1} c=-\Sigma(-1)^{\operatorname{deg} c_{i}^{\prime}}\left[s^{-1} c_{i}^{\prime}, s^{-1} c_{i}^{\prime \prime}\right]
\end{aligned}
$$




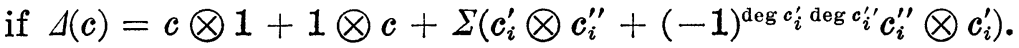

$\mathscr{L}(C)$ has a natural bigraded structure with generators $s^{-1} c$ having bigrading $=(-1$, degree $c)$.

REMARKS. $\mathscr{L}(C)=P \Omega C=$ primitive elements in the cobar construction $\Omega C$ an $C$. [1], [10]. Hence, $H \mathscr{L}(C)=H P \Omega C=P H \Omega C=P$ $\operatorname{Cotor}_{C}(k, k)$ where Cotor is the differential Cotor of Eilenberg and Moore [5]. That $H P \Omega C=P H \Omega C$ follows from the fact that $\Omega C$ is a cocommutative differential Hopf algebra. See Smith [25, p. 130] or Quillen [23, p. 280].

As a coalgebra, $\mathscr{C}(L)=S^{\prime}[s L]=$ the symmetric coalgebra on the suspension of $L$. The differential on $\mathscr{C}(L)$ splits as a sum of two differentials $d=d_{I}+d_{[]}$. A differential $d$ on $S^{\prime}[s L]$ is uniquely characterized by the projection

$$
\bar{d}: S^{\prime}[s L] \longrightarrow S^{\prime}[s L] \longrightarrow s L \text {. }
$$

(See 1.6 and 1.9.)

With respect to the natural generators $s \ell,\left(s \ell^{\prime \prime}\right)\left(s \ell^{\prime \prime}\right)=s \ell^{\prime} \otimes$ $s \ell^{\prime \prime}+(-1)^{\operatorname{deg} \ell^{\prime} \operatorname{deg} \ell^{\prime \prime}} s \ell^{\prime \prime} \otimes s \ell^{\prime}, \cdots$ of $S^{\prime}[s L] \subset T^{\prime}[s L]$,

$$
\begin{aligned}
& \bar{d}_{I}(s \ell)=-s d \ell \\
& \bar{d}_{[]}\left(s \ell^{\prime}\right)\left(s \ell^{\prime \prime}\right)=s\left[\ell^{\prime}, \ell^{\prime \prime}\right]
\end{aligned}
$$

and are zero on all other generators.

$\mathscr{C}(L)$ has a natural bigraded structure with elements $s \ell$ having bigrading $=(1$, degree $\ell)$.

REMARK. If $L$ is concentrated in degree 0 , then $\mathscr{C}(L)$ is just the standard complex for calculating Lie algebra homology. In general, $\mathscr{C}(L)$ is a subcoalgebra of $B U(L)=$ the bar construction on the universal enveloping algebra of $L$ and the injection $\mathscr{C}(L) \rightarrow$ $B U(L)$ is a homology isomorphism [17]. Hence, $H \mathscr{C}(L)=\operatorname{Tor}_{U[L]}(k, k)$ where this is the differential Tor of Eilenberg and Moore [25].

The adjunction maps $\alpha: \mathscr{L} \mathscr{C}(L) \rightarrow L$ and $\beta: C \rightarrow \mathscr{C} \mathscr{L}(C)$ may be described as follows:

(a) $\alpha$ is the unique map extending $s^{-1} \overline{\mathscr{C}}(s L) \rightarrow \mathscr{C}(s L) \rightarrow s L \rightarrow L$,

(b) $\beta$ is the unique map lifting $C \rightarrow s^{-1} \bar{C} \rightarrow \mathscr{L}(C) \rightarrow s \mathscr{L}(C)$.

Proposition 4.1. The adjunction maps $\mathscr{L} \mathscr{C}(L) \rightarrow L$ and $C \rightarrow$ $\mathscr{C} \mathscr{L}(C)$ are homology isomorphisms.

This is an immediate consequence of our next theorem.

Proposition 4.2. (a) Let $C$ be a coalgebra such that $C^{\sharp}=S^{\prime}[V]$, 
then $s^{-1} H P C$ is isomorphic to $H \mathscr{L}(C)$ as Lie algebras.

(b) Let $L$ be a Lie algebra such that $L^{\sharp}=F[V]$, then $s H Q L$ $i$ isomorphic to $\bar{H} \mathscr{C}(L)$ as coalgebras.

Proof. (a) Consider the map $s^{-1} P C \rightarrow \mathscr{L}(C)$. Filter $\mathscr{L}(C)$ by the second coordinate of its bidegree and give $s^{-1} P C$ a trivial one stage filtration. The map is a map of filtered objects and both filtrations are bicomplete [7]. Hence, a spectral sequence isomorphism implies a homology isomorphism $s^{-1} P C \rightarrow \mathscr{L}(C)$.

In the spectral sequence for $s^{-1} P C$ we have $E^{1}=s^{-1} P C, E^{2}=$ $H\left(s^{-1} P C\right)$.

In the spectral sequence for $\mathscr{L}(C)$, we have $E^{0}=\mathscr{L}\left(C^{\sharp}\right)=$ $P \Omega\left(C^{\sharp}\right) . \quad E^{1}=H P \Omega\left(C^{\sharp}\right)=P H \Omega\left(C^{\sharp}\right)=P S^{\prime}\left[s^{-1} V\right]=s^{-1} P C$.

$E^{2}=H\left(s^{-1} P C\right)$. Hence, $s^{-1} H P C \rightarrow H \mathscr{L}(C)$ is an isomorphism of graded vector spaces. That it is an isomorphism of Lie algebra follows from

$$
\begin{aligned}
d s^{-1}(x \otimes & \left.\otimes+(-1)^{\operatorname{deg} x \operatorname{deg} y} y \otimes x\right) \\
= & -s^{-1} d\left(x \otimes y+(-1)^{\operatorname{deg} x \operatorname{deg} y} y \otimes x\right) \\
& -(-1)^{\operatorname{deg} x}\left[s^{-1} x, s^{-1} y\right]
\end{aligned}
$$

if $x$ and $y$ are primitive in $C$.

(b) Consider the map $\mathscr{C}(L) \rightarrow s Q L$. As before, filter $\mathscr{C}(L)$ by the second coordinate of its bidegree and give $s Q L$ a trivial one stage filtration. Both filtrations are bicomplete and it suffices to compute the spectral sequence for $\mathscr{C}(L)$.

$E^{0}=\mathscr{C}\left(L^{\sharp}\right)$. Since $L^{\sharp}=F[V]$ is free, $E^{1}=H \mathscr{C}\left(L^{\sharp}\right)=\operatorname{Tor}_{U(L \sharp)}(k, k)=$ $k \oplus s V=k \oplus s Q L$.

Hence, $\bar{H} \mathscr{C}(L) \rightarrow s H Q L$ is a isomorphism of vector spaces. That it is an isomorphism of coalgebras follows from the observation that: If $\ell \in L$ projects to a cycle in $Q(L)$ and if $s \zeta+\Sigma\left(s \ell^{\prime}\right)\left(s \ell^{\prime \prime}\right)+\cdots$ is a cycle in $\mathscr{C}(L)$, then $-d \ell+\Sigma\left[\ell^{\prime}, \ell^{\prime \prime}\right] \equiv 0$ modulo boundaries and elements of length $\geqq 3$ in $L$.

The next proposition is an immediate consequence of the definitions of $\mathscr{C}$ and $\mathscr{L}$.

Proposition 4.3. (a) If $L$ is a Lie algebra, then $s^{-1} H P \mathscr{C}(L)=$ $H L$ as Lie algebras.

(b) If $C$ is a coalgebra, then $s H Q \mathscr{L}(C)=\bar{H} C$ as coalgebras.

We close this section with:

Proposition 4.4. (a) If $L \rightarrow L^{\prime}$ is a homology isomorphism of Lie algebras, then $\mathscr{C}(L) \rightarrow \mathscr{C}\left(L^{\prime}\right)$ is a homology isomorphism. 
(b) If $C \rightarrow C^{\prime}$ is a homology isomorphism between simply connected coalgebras, then $\mathscr{L}(C) \rightarrow \mathscr{L}\left(C^{\prime}\right)$ is a homology isomorphism.

Proof. Both (a) and (b) are consequences of respective EilenbergMoore spectral sequences. (Filter by the first coordinate of the bidegree.)

In (a), $E^{2}=H \mathscr{C}(H L)$ and the filtration is bicomplete, so we have no problems [7].

In (b), $E^{2}=H \mathscr{L}(H C)$ and the filtration is bicomplete if $C$ is simply connected.

The simply connected hypothesis is necessary in $4.4 \mathrm{~b}$. For example, let $C=\mathscr{C}$ (so (3)) where so (3) is the real Lie algebra of the Lie group SO (3). (We regard so (3) as being concentrated in dimension zero.) A classical computation of $E$. Cartan (see H. Weyl's book [29]) states that $H C=H \mathscr{C}($ so $(3))=H(\mathrm{SO}(3) ; R)=S^{\prime}[x]$ with degree $x=3$. Hence, there is a homology isomorphism $C \rightarrow C^{\prime}$ where $C^{\prime}=S^{\prime}[x]$. Then $H \mathscr{L}(C)=$ so (3) by 4.1 , but $H \mathscr{L}\left(C^{\prime}\right)=F\left[s^{-1} x\right]$ by 4.2. Therefore, $\mathscr{L}(C) \rightarrow \mathscr{L}\left(C^{\prime}\right)$ is not a homology isomorphism.

In 6.4, we shall draw the conclusion of $4.4 \mathrm{~b}$ with an alternate hypothesis.

5. Cofibrant and fibrant models. We begin this section by observing that $D G L A, D G A$, and $D G C$ are closed model categories in the sense of Quillen [22] [23]. Recall Quillen's definition.

DEFINITION 5.1. A closed model category is a category $\mathscr{C}$ endowed with three selected classes of morphisms, called fibrations, cofibrations, and weak equivalences and subject to the following axioms:

$C M$ 1. $\mathscr{C}$ is closed under finite direct and inverse limits.

$C M$ 2. If $g f$ is defined and any two of $f, g, g f$ are weak equivalences, then so is the third.

$C M$ 3. A retract of a fibration, cofibration, or weak equivalence in one also.

$C M$ 4. We can complete any commutative diagram

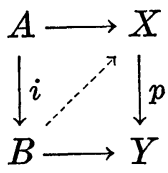


whenever $i$ is a cofibration, $p$ is a fibration, and either $p$ or $i$ is a weak equivalence.

$C M$ 5. Any map $f$ may be factored in two ways: $f=p i$ where $p$ is a fibration, $i$ is a cofibration, and either $p$ or $i$ is a weak equivalence.

Following Quillen [23], DLGA is a closed model category with weak equivalences being maps which induce isomorphisms in homology, fibrations being maps which are surjective, and cofibrations being maps which satisfy axiom $C M 4$ for all fibrations which are weak equivalences. Although Quillen treats only those Lie algebras with $L_{0}=0$ and uses a slightly different notion of fibration, his proof that they form a closed model category remains valid in this more general context.

Bousfield and Gugenheim [2] have shown that $D G A$ is a closed model category with weak equivalences being maps which induce isomorphisms in cohomology, fibrations being maps which are surjective, and cofibrations being maps which satisfy axiom $C M 4$ for all fibrations which are weak equivalences.

Again following Quillen [23], DGC is a closed model category with weak equivalences being maps which induce isomorphisms in homology, cofibrations being maps which are injective, and fibrations being maps which satisfy axiom $C M 4$ for all cofibrations which are weak equivalences. Unfortunately, Quillen's proof that this is a closed model category [23, p. 256] relies heavily upon the application of the comparison theorem to spectral sequences which happen to have a sufficiently nice $E^{2}$ term only when the coalgebras involved are simply connected, $C_{1}=0$. Fortunately, a proof can be given which avoids these technical difficulties.

Proposition 5.2. DGC (DGA, DGLA) is a closed model category.

Proof. Only axioms $C M 4$ and $C M 5$ are nontrivial and half of $C M 4$ is true by definition.

In the course of this proof, we need two kinds of standard fibrations.

Lemma 5.3. (a) If $V$ is a $D G$ vector space, then $S^{\prime}[V] \rightarrow k$ is a fibration.

(b) If $V_{n}$ is concentrated in dimension $n \geqq 1$, then $E^{\prime}\left[V_{n}\right] \rightarrow$ $S^{\prime}\left[V_{n}\right]$, which is defined in $\S 3$, is a fibration.

The proof of 5.3 is an exercise in the universal mapping property of symmetric coalgebras. Since pullbacks of fibrations are fibrations, 
5.3b implies that elementary extensions (3.7) are fibrations.

Now let $f: C \rightarrow D$ be a map in $D G C$. We will verify $C M 5$ for this map:

(a) $f=p i$ where $p$ is a weak equivalence.

(b) $f=p i$ where $i$ is a weak equivalence.

(a) Pick an acyclic $D G$ vector space $V, H V=0$, and an injective $D G$ map $C \rightarrow V$. By the universal property of $S^{\prime}$, there is a unique factorization $C \rightarrow S^{\prime}[V] \rightarrow V$ and $S^{\prime}[V]$ is a acyclic by 1.10 . Since $\otimes$ is the product in $D G C$, there is an injective map $i: C \rightarrow$ $D \otimes S^{\prime}[V]$ and the projection $p: D \otimes S^{\prime}[V] \rightarrow D$ is a weak equivalence. $p$ is a fibration since it is the pullback of the fibration $S^{\prime}[V] \rightarrow k$ under $D \rightarrow k$.

(b) By part (a), we can assume that $C \rightarrow D$ is an injection. Pick a $D G$ map $C \rightarrow H C$ which is a homology isomorphism. This need not be a coalgebra map. This defines $C \rightarrow S^{\prime}[H C]$ and, by replacing $C \rightarrow D$ by $C \rightarrow D \otimes S^{\prime}[H C]$, we may assume that $C \rightarrow D$ is both injective and injective on homology.

Make the inductive assumption that we have constructed a factorization $C \underset{i_{n}}{\rightarrow} E_{n} \underset{p_{n}}{\rightarrow} D$ where $p_{n}$ is a fibration, $i_{n}$ is a cofibration, and $H\left(i_{n}\right)$ is an isomorphism in dimensions $\leqq n$.

Pick a projection $E_{n} \rightarrow$ coker $H_{n+1}\left(i_{n}\right)$. This defines a map $E_{n} \rightarrow$ $S^{\prime}$ [coker $\left.H_{n+1}\left(i_{n}\right)\right]$ and it is not hard to see that there is a commutative diagram

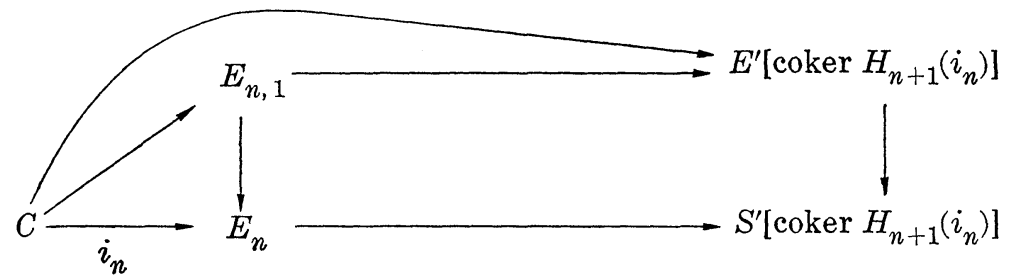

where $E_{n, 1} \rightarrow E_{n}$ is the pullback fibration.

Repeating this process, construct an inverse system of fibrations $\cdots \rightarrow E_{n, 2} \rightarrow E_{n, 1} \rightarrow E_{n}$.

Set $E_{n+1}=\underset{\leftarrow}{\lim } E_{n, m}$ and let $i_{n+1}: C \rightarrow E_{n+1}$ be the natural map. Define $p_{n+1}$ to be the composition $E_{n+1} \rightarrow E_{n} \rightarrow D$. Then $i_{n+1}$ is a cofibration and $p_{n+1}$ is a fibration since it is a composition of fibrations. Finally, $H\left(i_{n+1}\right)$ is an isomorphism in dimensions $\leqq n+1$. This verifies the inductive assumption.

Set $E=\lim _{\leftarrow} E_{n}$. The factorization $C \rightarrow E \rightarrow D$ verifies the second half of $C M 5$.

To complete the verification that $D G C$ is a closed model cate- 
gory, it remains to demonstrate $C M 4$ when $p: X \rightarrow Y$ is a fibration and a weak equivalence. In verifying the first half of $C M 5$ we showed that $p$ could be factored as $X \underset{i}{\overrightarrow{1}} Y \otimes S^{\prime}[V] \underset{q}{\rightarrow} Y$ where $i$ is a cofibration and $p$ is a fibration and weak equivalence. By $C M 2$, $i$ is a weak equivalence. By the definition of a fibration, we can complete the commutative diagram below.

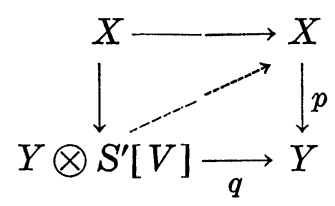

Hence, $p$ is a retract of the product fibration $q$. It is easy to verify $C M 4$ for $q$ and hence for $p$.

This completes the proof that $D G C$ is a closed model category.

Given a pointed closed model category $\mathscr{C}$ with point object *, an object $X$ is called cofibrant (respectively, fibrant) if the map $* \rightarrow X$ is a cofibration (resp., if $X \rightarrow^{*}$ is a fibration). Notice that $D G L A, D G C$, and $D G A$ are pointed with point objects $0, k$, and $k$, respectively. In $D G L A$ and $D G A$, every object is fibrant and in $D G C$ every object in cofibrant.

Definition 5.4. Given an object $X$ in a pointed closed model category, a cofibrant model (respectively, fibrant model) for $X$ is a cofibrant (fibrant) object $Y$ and a weak equivalence $Y \rightarrow X(X \rightarrow Y)$.

Cofibrant and fibrant models always exist because of axiom $C M$ 5. We shall adopt the notation $c X \rightarrow X$ for cofibrant models and $X \rightarrow f X$ for fibrant models. Notice that $C M 5$ implies that we may take $c X \rightarrow X$ to be a fibration and $X \rightarrow f X$ to be a cofibration. Using $C M 4$, it is easy to see that $c$ (and $f$ ) give well defined functors from $\mathscr{C}$ to the homotopy category ho $\mathscr{C}_{c}$ of cofibrant (ho $\mathscr{C}_{f}$ of fibrant) objects of $\mathscr{C}$ [22].

(Given a category $\mathscr{C}$ and a multiplicative class of morphisms $S$ in $\mathscr{C}$, in our case weak equivalences, the homotopy category ho $\mathscr{C}=$ $S^{-1} \mathscr{C}$ is the localization of $\mathscr{C}$ with respect to $S$ [9]).

Definition 5.5. An algebra $A$ (respectively, Lie algebra $L$ ) is called minimal if it is cofibrant and the induced differential on $Q(A)$ (respectively, $Q(L)$ ) is zero. A coalgebra $C$ is called minimal if it is fibrant and the differential is zero on $P(C)$.

A minimal model for an algebra $A$, denoted by $m A \rightarrow A$, or for a Lie algebra $L$, denoted by $m L \rightarrow L$, is a minimal cofibrant model. A minimal model for a coalgebra $C$, denoted by $C \rightarrow m C$, is a minimal 
fibrant model.

The rest of this section will be devoted to the following five propositions, 5.6-5.10 Taken together, they give a rather complete characterization of cofibrant, fibrant, and minimal models.

Proposition 5.6. (a) Every algebra $A$ has a minimal model $m A \rightarrow A$ ond $m A$ is unique up to isomorphism.

(b) Every coalgebra $C$ has a minimal model $C \rightarrow m C$ and $m C$ is unique up to isomorphism.

(c) Every Lie algebra $L$ with $L_{0}=0$ has a minimal model $m L \rightarrow L$ and $m L$ is unique up to isomorphism.

Although it is not true that only those Lie algebras $L$ with $L_{0}=0$ have minimal models, it is easy to see that some restriction is necessary. For example, let $L=$ so $(3)=$ the real Lie algebra of the Lie group SO (3). Suppose $m L \rightarrow L$ were a minimal model. Then $Q(m L)_{0} \neq 0$ since $H_{0} L=$ so $(3) \neq 0$. But $4.2 \mathrm{~b}$ and $4.4 \mathrm{a}$ imply that $Q(m L)_{0}=H_{1} \mathscr{C}(m L)=H_{1} \mathscr{C}(L)=0$ since $H \mathscr{C}(L)=H(\mathrm{SO}(3) ; R)=$ $H\left(S^{3} ; R\right)$ [29]. Hence, no minimal model for so (3) exists.

Conjecture. A Lie algebra $L$ with $H L$ nilpotent has a minimal model.

Proposition 5.7. (a) An algebra $A$ is cofibrant if and only if $A \approx m A \otimes S[V]$ where $H V=0$.

(b) A coalgebra $C$ is fibrant if and only if $C \approx m C \otimes S^{\prime}[V]$ where $H V=0$.

(c) A Lie algebra is cofibrant if and only if it is a retract of a Lie algebra $L$ with $L^{\#}=a$ free Lie algebra.

Proposition 5.8. (a) An algebra $A$ is minimal if and only if $A$ is constructible by decomposable elementary extensions starting from the ground field $k$.

(b) A coalgebra $C$ is minimal if and only if $C$ is constructible by nonprimitive elementary extensions starting from the ground field $k$.

(See 3.9 for the definition of constructible by elementary extensions. For algebras, replace inverse limits by direct limits in the definition for coalgebras.)

Note that if we combine $5.8 \mathrm{~b}$ with 3.11 , we see that a coalgebra $C$ is minimal if and only if $C$ is a reduced symmetric coalgebra with the Lie algebra $s^{-1} H P C=s^{-1} P C$ a nilpotent completion. 
Proposition 5.9. A coalgebra $C$ is fibrant if and only if $C^{\sharp}=$ a symmetric coalgebra and the Lie algebra $s^{-1} H P C$ is a nilpotent completion.

Proposition 5.10. If $A$ is cofibrant (respectively, minimal) algebra of finite type, then $A^{*}$ is a fibrant (reespctively, minimal) coalgebra.

REMARK. By 5.7, and 5.8, cofibrant algebras are symmetric algebras, fibrant coalgebras are symmetric coalgebras, and cofibrant lie algebras are free Lie algebras.

The results 5.6, 5.7 and 5.8 are known for algebras. See [4] and [2]. Furthermore, 5.7 and 5.8 imply 5.10 since the dual of a decomposable elementary extension is a nonprimitive elementary extension and the dual of a symmetric algebra is a symmetric coalgebra. It remains to show 5.6, and 5.7, 5.8, and 5.9 for coalgebras and Lie algebras.

We treat coalgebras first. A slight modification of the proof of $C m 5 \mathrm{~b}$ in 5.2 shows that we can factor $C \rightarrow k$ into $C \rightarrow D \rightarrow k$ where $C \rightarrow D$ is a weak equivalence (but not a cofibration) and $D \rightarrow k$ is a fibration. Given $C \rightarrow D_{n} \rightarrow k$ where $D_{n} \rightarrow k$ is a fibration and $H C \rightarrow$ $H D_{n}$ is an isomorphism in dimensions $\leqq n$ and an epimorphism in dimension $n+1$, construct a nonprimitive elementary extension $D_{n}^{\prime} \rightarrow$ $D_{n}$ of dimension $n+1$ and a factorization $C \rightarrow D_{n}^{\prime} \rightarrow D_{n}$ such that $H C \rightarrow H D_{n}^{\prime}$ is an isomorphism in dimensions $\leqq n+1$. Specifically, $D_{n}^{\prime}=D_{n} \otimes S^{\prime}[V]$ where $V=$ kernel of $H_{n+1}\left(C \rightarrow D_{n}\right)$. Following the proof of 5.2, construct an inverse limit of nonprimitive elementary extensions $D_{n+1} \rightarrow D_{n}^{\prime}$ of dimension $n+2$ and a factorization $C \rightarrow$ $D_{n+1} \rightarrow D_{n}^{\prime}$ such that $H C \rightarrow H D_{n+1}$ is an isomorphism in dimensions $\leqq n+1$ and an epimorphism in dimension $n+2$. Then $D=\lim D_{n}$ yields a factorization $C \rightarrow D \rightarrow k$ with $D \rightarrow k$ a fibration and $\overleftarrow{C \rightarrow D}$ a weak equivalence. $D$ is therefore fibrant and, by the remark after $3.11, D$ is a reduced symmetric coalgebra, therefore minimal.

Hence, minimal models for coalgebras exist. Furthermore, the above minimal model $m C=D$ is constructible by nonprimitive elementary extensions starting from the ground field.

REMARK. The above procedure is dual to Sullivan's construction of minimal models for algebras.

Lemma 5.11. (a) If $X \rightarrow Y$ is a cofibration and a weak equivalence and $X$ is fibrant, then there is a splitting $Y \rightarrow X$ such that $X \rightarrow Y \rightarrow X$ is the identity.

(b) If $X \rightarrow Y$ is a fibration and a waak equivalence and $Y$ is 
cofibrant, then there is a splitting $Y \rightarrow X$ such that $X \rightarrow Y \rightarrow X$ is the identity.

This lemma is a consequence of axiom $C M 4$.

To show the uniqueness of minimal coalgebra models, let $C \rightarrow$ $m C$ be any minimal model and let $C \rightarrow m_{0} C$ be the specific minimal model constructed above. We can find a fibrant model $C \rightarrow m C \otimes$ $S^{\prime}[V]$ such that this map is a cofibration and $H V=0$. CM 4 implies that there is a map $m C \otimes S^{\prime}[V] \rightarrow m_{0} C$ which is a weak equivalence. Hence, the composition $m C \rightarrow m C \otimes S^{\prime}[V] \rightarrow m_{0} C$ is a weak equivalence between minimal coalgebras. We claim it is an isomorphism.

Factor $m C \rightarrow m_{0} C$ into $m C \rightarrow m_{0} C \otimes S^{\prime}[W] \rightarrow m_{0} C$ where $m C \rightarrow$ $m_{0} C \otimes S^{\prime}[W]$ is a cofibration and $H W=0$. Apply 5.11a to get a splitting $m_{0} C \otimes S^{\prime}[W] \rightarrow m C . \quad m C$ is a retract of a symmetric coalgebra, therefore, it is a symmetric coalgebra. Hence, $P(m C)=$ $H P(m C) \rightarrow H P\left(m_{\mathrm{f}} C \otimes S^{\prime}[V]\right)=P\left(m_{0} C\right)$ is a split monomorphism with splitting $P\left(m_{0} C\right) \rightarrow P(m C)$ induced by $m_{0} C \otimes S^{\prime}[W] \rightarrow m C$.

Replacing $m C \rightarrow m_{0} C$ by $m_{0} C \otimes S^{\prime}[W] \rightarrow m C$ in the above paragraph, we conclude that $P\left(m_{0} C\right) \rightarrow P(m C)$ is also a split monomorphism. Hence, $P(m C) \rightarrow P\left(m_{0} C\right)$ is an isomorphism. Since $m C$ and $m_{0} C$ are symmetric coalgebras, $m C \rightarrow m_{0} C$ is an isomorphism. This concludes the proof of 5.6 for coalgebras.

The minimal model $m_{0} C$ is constructible by nonprimitive elementary extensions starting from the ground field. Any coalgebra so constructible is minimal. Together with 5.6, this proves 5.8 for coalgebras.

Since the minimal coalgebra $m_{0} C$ satisfies 5.9, uniqueness proves 5.9 for all minimal coalgebras. An argument similar to the proof of 3.12 proves 5.7 for coalgebras. Proposition 5.9 for fibrant coalgebras follows from 5.7 and 5.9 for minimal coalgebras. This completes the proofs for coalgebras.

It remains to prove 5.6 and 5.7 for Lie algebras.

It is easy to see that any Lie algebra $L$ with $L^{\sharp}=$ a free Lie algebra is cofibrant. Hence, so is any retract. Conversely, Quillen has shown [23, p. 256] that any cofibrant Lie algebra $L$ is a retract of a Lie algebra $L^{\prime}$ with $\left(L^{\prime}\right)^{\#}=$ a free Lie algebra. His proof remains valid if $L_{0} \neq 0$. This proves 5.7 for Lie algebras.

Let $L$ be a Lie algebra with $L_{0}=0$. Pick a chain splitting $H_{1} L \rightarrow L$. This defines a map $F_{1}=F\left[H_{1} L\right] \rightarrow L$ which is an epimorphism in one dimensional homology. $F_{1}$ is minimal.

Inductively, suppose we are given $F_{n} \rightarrow L$ which is a isomorphism (epimorphism) in homology in dimensions $<n(<n+1)$ and such that $F_{n}$ is minimal and free on generators concentrated in dimensions $<n+1$. Furthermore, suppose $F_{n}=F[Z]$ with a splitting $Z_{n}=$ 
$W_{n} \oplus V_{n}$ where $d\left(W_{n}\right)=0$, and $d: V_{n} \rightarrow H F_{n-1}$ and $W_{n} \rightarrow H F_{n} \rightarrow H L$ are monomorphisms. Construct $F_{n+1} \rightarrow L$ as follows. Pick a lift $V \rightarrow F_{n}$ where $V=\operatorname{ker} H_{n}\left(F_{n} \rightarrow L\right)$. Consider the composition $d$ : $s V \rightarrow V \rightarrow F_{n}$ and use this to extend the differential on $F_{n}$ to $G_{n+1}=F_{n} \vee F[s V]$. Then $G_{n+1}$ is minimal and maps isomorphically in homology in dimension $<n+1$.

Let $W=$ coker $H_{n+1}\left(G_{n+1} \rightarrow L\right)$ and pick a splitting $W \rightarrow L$. Define $d$ to be zero on $W$ and use this to extend the differential on $G_{n+1}$ to $F_{n+1}=G_{n+1} \vee F[W]$. Then $F_{n+1}$ is minimal and $W \rightarrow L$ defines and extension to a map $F_{n+1} \rightarrow L$ which is an isomorphism (epimorphism) in homology in dimensions $<n+1(<n+2)$. The required splitting of $F_{n+1}$ is obvious from our construction.

Setting $F=\lim _{n} F_{n}$, the map $F \rightarrow L$ is a weak equivalence and $F$ is a minimal model for $L$.

Finally, minimal Lie algebra models are unique up to isomorphism by an argument similar to that for the uniqueness of minimal coalgebra models. This completes the proof of 5.6 for Lie algebras.

6. Homotopy groups. We now define "homotopy" groups for Lie algebras, coalgebras, and algebras.

Definition 6.1. (a) If $L$ is a Lie algebra, then $\pi L=H Q(c L)$.

(b) If $C$ is a coalgebra, then $\pi C=H P(f c)$.

(c) If $A$ is an algebra, then $\pi A=H Q(c A)$.

That $\pi C$ and $\pi A$ are well defined up to isomorphism follows from 5.6 and 5.7. That $\pi L$ is well defined up to isomorphism will follow from 6.3. If we choose minimal models, then $\pi L=Q(m L), \pi C=$ $P(m C)$, and $\pi A=Q(m A)$.

By $\S 2, s \pi L$ has a coalgebra structure, $s^{-1} \pi C$ has a Lie algebra structure, and $s^{-1} \pi A$ has a Lie coalgebra structure. As a consequence of 5.9, we have:

Proposition 6.2. For any coalgebra $C$, the Lie algebra $s^{-1} \pi C$ is a nilpotent completion.

From 4.2 and 4.4 we get:

Proposition 6.3. (a) If $L$ is a Lie algebra, then $s \pi L=H \mathscr{C}(L)=$ $H \mathscr{C}(c L)$ as coalgebras.

(b) If $C$ is a coalgebra, then $s^{-1} \pi C=H \mathscr{L}(f C)$ as Lie algebras. If $C_{1}=0$, then $s^{-1} \pi C=H \mathscr{L}(C)$.

REMARK. This result shows that $\pi L$ is a functor and $\pi C$ is a functor if $C_{1}=0$. Bousfield and Gugenheim [2] have shown that $\pi A$ 
is a functor by constructing a natural cofibrant model $c A \rightarrow A$. It is possible to construct natural cofibrant (fibrant) models $c L \rightarrow L(C \rightarrow f C)$. Hence, $\pi L, \pi C, \pi A$ are functors with no connectivity hypothesis.

Since $\pi C$ is well defined, 6.3 implies:

Proposition 6.4. If $f: C \rightarrow C^{\prime}$ is a weak equivalence between fibrant coalgebras, then $\mathscr{L}(f): \mathscr{L}(C) \rightarrow \mathscr{L}\left(C^{\prime}\right)$ is a weak equivalence.

Together with $4.4 \mathrm{~b}, 6.3$ implies:

Proposition 6.5. If $C$ is a coalgebra with $C_{1}=0$, then $s^{-1} \pi C=$ $H \mathscr{L}(C)$.

7. Equivalence of homotopy categories. Recall Quillen's equivalence theorem [23, p. 235] and [22, 4].

THEOREM 7.1. Let $\mathscr{C}_{1}$ and $\mathscr{C}_{2}$ be closed model categories and let

$$
\mathscr{C}_{1} \underset{G}{\stackrel{F}{\rightleftarrows}} \mathscr{C}_{2}
$$

be a pair of adjoint functors such that

(1) $F$ preserves cofibrations and $G$ preserves fibrations.

(2) If $f: A \rightarrow B$ is a weak equivalence between cofibrant objects in $\mathscr{C}_{1}$, then $F(f)$ is a weak equivalence in $\mathscr{C}_{2}$. If $g: X \rightarrow Y$ is a weak equivalence between fibrant objects in $\mathscr{C}_{2}$, then $G(g)$ is a weak equivalence in $\mathscr{C}_{1}$.

(3) If $A$ is a cofibrant object in $\mathscr{C}_{1}$ and $X$ is a fibrant object in $\mathscr{C}_{2}$, then a map $f: A \rightarrow G X$ is a weak equivalence if and only if the corresponding map $f^{b}: F A \rightarrow X$ is a weak equivalence.

Then $F$ and $G$ are induce equivalences of homotopy categories. Moreover, if $\mathscr{C}_{1}$ and $\mathscr{C}_{2}$ are pointed, then this equivalence preserves the loop and suspension functors and the families of fibration and cofibration sequences.

A careful reading of Quillen's proof shows that $F$ and $G$ induce equivalences of homotopy categories even if hypothesis 1 is weakened to: $F$ preserves cofibrant objects and $G$ preserves fibrant objects. (See [22], 4.7, (3).)

Let $n c D G L A$ be the full subcategory of $D G L A$ consisting of Lie algebras $L$ such that $H L$ is a nilpotent completion.

Unfortunately, ncDGLA is not a closed model category since it is not closed under finite coproducts. Hence, the homotopy category, $h o(n c D G L A)$, does not make sense by itself. What we mean by $h o(D G L A)$ is the full subcategory of $h o(D G L A)$ generated by objects 
of neDGLA. As in the paper [2] of Bousfield and Gugenheim, we shall use this convention whenever we are dealing with a full subcategory of a closed model category.

Proposition 7.2. The adjoint functors

$$
D G C \underset{\mathscr{C}}{\stackrel{\mathscr{L}}{\rightleftarrows}} D G L A
$$

induce equivalence of homotopy categories ho $(D G C) \approx h o(n c D G L A)$.

Proof. Since $\mathscr{L}(C)^{\#}=$ a free lie algebra, $\mathscr{L}(C)$ is always cofibrant by 5.7. Hence, $\mathscr{L}$ preserves cofibrant objects. If $L$ is an object in $n c D G L A$, then $\mathscr{C}(L)^{*}=$ a symmetric coalgebra with $s^{-1} H P \mathscr{C}(L)=H L$, a nilpotent completion. Therefore, $\mathscr{C}(L)$ is fibrant by 5.9 and $\mathscr{C}$ preserves fibrant objects when restricted to objects in $n c D G L A$.

Hypothesis 2 in 7.1 is a consequence of 4.4 and 6.4. Hypothesis 3 follows from hypotheses 1 and 2 and 4.1.

Hence, 7.1 implies 7.2. Specifically, the equivalence of homotopy categories is given on objects by $C \rightarrow \mathscr{L}(f C)$ and $L \rightarrow \mathscr{C}(L)$. Notice that $H \mathscr{L}(f C)=s^{-1} H P(f C)$ by 4.2 and is a nilpotent completion by 5.9 .

In the remainder of this section, the ground field will be the rationals. We are going to relate the algebra developed in this paper to rational homotopy theory.

A connected pointed Kan complex, hereafter referred to as a space, is called nilpotent if $\pi_{1}$ is a nilpotent group and $\pi_{n}$ for all $n \geqq 2$ is a nilpotent $\pi_{1}$ module [2]. A nilpotent space $X$ is called rational if the groups $H_{n}(X ; Z)$, or equivalently the groups $\pi_{n}(X)$, are uniquely divisible. A nilpotent space $X$ is of finite $Q$-type if the vector spaces $H_{n}(X ; Q)$, and hence $\pi_{n}(X) \otimes \boldsymbol{Q}$ for $n \geqq 2$, are finite dimensional [2].

The homotopy category of rational nilpotent spaces is a retract of the homotopy category of nilpotent spaces. The retraction functor is denoted $X \rightarrow X \otimes \boldsymbol{Q}$ and if $X \otimes \boldsymbol{Q} \cong Y \otimes \boldsymbol{Q}$ we say that $X$ and $Y$ have the same rational homotopy type. (For details, see [8], [4], [3], or [23].)

Sullivan [4] [8] and, in a more functorial manner, Bousfield and Gugenheim [2] have shown that the homotopy category of rational finite $\boldsymbol{Q}$-type nilpotent spaces is equivalent to the homotopy category of rational algebras $A$ with $\pi A$ of finite type and $H^{\circ}(A)=\boldsymbol{Q}$.

We adopt the following notation:

(a) $S$ is the category of connected spaces. 
(b) $n S$ is the category of connected finite $\boldsymbol{Q}$-type nilpotent spaces.

(c) $S \otimes \boldsymbol{Q}$ is the category of rational spaces in $n S$.

(d) $n D G A$ is the full subcategory of $D G A$ of algebras $A$ with $\pi A$ of finite type.

(e) $n D G C$ is the full subcategory of $D G C$ of coalgebras $C$ with $\pi C$ of finite type.

(f) $n D G L A$ is the full subcategory of $D G L A$ of Lie algebras $L$ with $H L$ nilpotent of finite type.

(g) $\mathscr{E}: S \rightarrow D G A$ is Sullivan's $P L$ deRham functor (see [4] or [2]). [2].

(h) $R: D G A \rightarrow S$ is Bousfield and Gugenheim's realization functor

Proposition 7.3. The following four homotopy categories are equivalent: $h_{0}(S \otimes \boldsymbol{Q}), h o(n D G A), h_{0}(n D G C)$, and $h_{0}(n D G L A)$.

Proof. The equivalence of $h o(S \otimes \boldsymbol{Q})$ and $h o(n D G A)$ is due to Bousfield and Gugenheim and is given by the contravariant functors $X \rightarrow \mathscr{E}(X)$ and $A \rightarrow R(m A)$ [2].

The equivalence of $h o(n(D G A)$ and $h o(n D G C)$ is given by the contravariant functors $A \rightarrow(m A)^{*}$ and $C \rightarrow(m C)^{*}$.

The equivalence of $h o(n D G C)$ and $h o(n D G L A)$ is given by the restriction of the covariant functors in 7.2, i.e., $C \rightarrow \mathscr{L}(f C), L \rightarrow$ $\mathscr{C}(L)$. Note that, if $s^{-1} \pi C$ is of finite type, it is nilpotent since it is a nilpotent completion 6.2. Since $s^{-1} \pi C=H \mathscr{L}(f C)$ by 6.3 , $\mathscr{L}(f C)$ is in $n D G L A$. Conversely, given $L$ in $n D G L A, \mathscr{C}(L)$ is in $n D G C$ since $s^{-1} \pi \mathscr{C}(L)=H L$ by 4.3 .

8. Minimal models for spaces. Let $X$ be a connected finite $Q$-type nilpotent space. Using the equivalence in 7.3 , we may associate to $X$ a minimal algebra $M_{X}$, a minimal coalgebra $C_{X}$, and a cofibrant Lie algebra $\mathscr{L}\left(C_{X}\right)$. If $X$ is simply connected, we may associate to $X$ a minimal Lie algebra $L_{X}$. These three minimal objects are determined by $X$ up to isomorphism. They are called the minimal algebra model, the minimal coalgebra model, and the minimal Lie algebra model, respectively.

Specifically, $M_{X}=m \mathscr{E}(X)=$ the minimal algebra model for the $P L$ deRham forms. (This is contained in [4].) $C_{X}=\left(M_{X}\right)^{*}=$ the dual coalgebra to $M_{X}$ and $L_{X}=m \mathscr{L}\left(C_{X}\right)=$ the minimal Lie algebra model for $\mathscr{L}\left(C_{X}\right)$. We do not know whether $L_{X}$ always exists if $X$ is not simply connected. The following proposition is immediate.

Proposition 8.1. If $X$ and $Y$ are connected finite Q-type nilpotent spaces, then the following are equivalent: 
(a) $X \otimes Q \cong Y \otimes Q$.

(b) $M_{X}$ and $M_{Y}$ are isomorphic algebras.

(c) $C_{X}$ and $C_{Y}$ are isomorphic coalgebras.

(d) There is a weak equivalence $\mathscr{L}\left(C_{X}\right) \rightarrow \mathscr{L}\left(C_{Y}\right)$.

(e) If $X$ and $Y$ happen to be simply connected, $L_{X}$ and $L_{Y}$ are isomorphic Lie algebras.

Given a nilpotent group $G$, there is a universal morphism $G \rightarrow$ $G \otimes Q$ of $G$ into a nilpotent uniquely divisible group $G \otimes Q$. This is called the Malcev completion of $G$ and agrees with the tensor product when $G$ is abelian [13] [23].

There is an equivalence between the category of nilpotent uniquely divisible groups $G$ and the, category of nilpotent rational Lie algebras $L$ (nongraded) given by $G \rightarrow L=P \widehat{Q}[G]=$ the primitives of the completion of the rational group algebra with respect to its augmentation ideal [12] [23]. Given $G$ denote the corresponding Lie algebra by $\ell(G)$.

Proposition 8.2. If $X$ is a connected finite Q-type nilpotent space, then the following Lie algebras are isomorphic: $\ell\left(\pi_{1}(X) \otimes \boldsymbol{Q}\right)=$ $\left(\pi^{1} M_{X}\right)^{*}=\pi_{1} C_{X}=H_{0} \mathscr{L}\left(C_{X}\right)$.

Proof. The first isomorphism is in [4]. The rest follows from 4.2 .

For $n, m \geqq 2$ the Whitehead product $\pi_{n}(X) \otimes \pi_{m}(X) \rightarrow \pi_{n+m-1}(X)$ corresponds to the Samelson product $\pi_{n-1}(\Omega X) \otimes \pi_{m-1}(\Omega X) \rightarrow \pi_{n+m-2}(\Omega X)$ under the isomorphism $\pi_{n}(X)=\pi_{n-1}(\Omega X)$. For $n \geqq 2$ the Samelson product makes the homotopy groups $\pi_{n-1}(\Omega X)$ into a graded Lie algebra. The next proposition is proved in the same way as 8.2 with a similar assist from [4].

Proposition 8.3. If $X$ is a connected finite Q-type nilpotent space, then for $n \geqq 2$ the following are isomorphisms of graded Whitehead algebras: $\pi_{n}(X) \otimes Q=\left(\pi^{n} M_{X}\right)^{*}=\pi_{n} C_{X}=H_{n-1} \mathscr{L}\left(C_{X}\right)\left(=H_{n-1} L_{X}\right.$ if $X$ is simply connected).

From 4.2, we also get:

Proposition 8.4. If $X$ is a connected finite Q-type nilpotent space, the following are isomorphisms of graded coalgebras: $H_{n}(X ; \boldsymbol{Q})=H^{n}\left(M_{X}\right)^{*}=H_{n}\left(C_{X}\right)=\pi_{n-1}\left(\mathscr{L}\left(C_{X}\right)\right)$.

Propositions 8.2, 8.3, and 8.4 give the following description of 
minimal models.

The minimal Lie algebra model $L_{X}$ is the free Lie algebra generated by $s^{-1} \bar{H}(X ; \boldsymbol{Q})$ with differential $d$ given on generators $s^{-1} x$ by $d s^{-1} x=\Sigma\left[s^{-1} a_{i}, s^{-1} b\right]+P$, where $P$ is a sum of brackets of length three or greater and $\Delta(x)=\Sigma a_{i} \otimes b_{i}+(-1)^{\operatorname{deg} a_{i} \operatorname{deg} b_{i}} b_{i} \otimes a_{i}$.

The minimal coalgebra model $C_{X}$ is the symmetric coalgebra with primitives equal to $\ell\left(\pi_{1}(X) \otimes Q\right)+\sum_{n \geqq 2} \pi_{n}(X) \otimes Q=s H L_{X}$.

The minimal algebra model $M_{X}$ is the symmetric algebra generated by the dual of $P C_{X}$ [4].

As exercises for the reader we leave the following lemmas.

LEMMA 8.5. $M_{X \times Y}=M_{X} \otimes M_{Y}, C_{X \times Y}=C_{X} \otimes C_{Y}$.

LEMMA 8.6. $L_{X \vee Y}=L_{X} \vee L_{Y}$ if $X$ and $Y$ are simply connected.

Recall Sullivan's definition of formality [4].

Definition 8.7. Let $X$ be a finite $Q$-type nilpotent space. $X$ is called formal if there is a weak equivalence $M_{X} \rightarrow H^{*}(X ; Q)$.

The next proposition enables one to construct the minimal Lie algebra model for many examples.

Proposition 8.8. If $X$ is formal and simply connected, then $L_{X}$ is isomorphic to $\mathscr{L} H(X ; Q)$.

Proof. Dualize $M_{X} \rightarrow H^{*}(X ; Q)$ to get a weak equivalence $H(X ; Q) \rightarrow C_{X}$. By $4.4 \mathrm{~b}, \mathscr{L} H(X ; Q) \rightarrow \mathscr{L}\left(C_{X}\right)$ is a weak equivalence since $C_{X}$ is simply connected. Since $H(X ; Q)$ has zero differential, $H(X ; Q)$ has a decomposable differential and is a minimal Lie algebra. Since minimal models are unique up to isomorphism, $L_{X}$ is isomorphic to $\mathscr{L} H(X ; Q)$.

For example, let $X=\mathrm{V} S^{n_{\alpha}}, n_{\alpha} \geqq 2$, be a wedge of spheres. Then $X$ is formal. (See, for example, [15].) Hence, $L_{X}$ is isomorphic to $\mathscr{L} H(X ; Q)$, the free Lie algebra on generators $x_{\alpha}$ with degree $x_{\alpha}=n_{\alpha}-1$. Since all nontrivial cup products vanish, $d x_{\alpha}=0$ for all $\alpha$.

$C P^{n}$ is formal. In fact, any compact Kahler manifold is formal [4]. Hence, $L_{C P^{n}}$ is isomorphic to $\mathscr{L} H\left(C P^{n} ; Q\right)$, the free Lie algebra generated by $\gamma_{1}, \cdots, \gamma_{n}$ with degree $\gamma_{k}=2 k-1$ and $d \gamma_{k}=$ $-1 / 2 \sum_{i+j=k}\left[\gamma_{i}, \gamma_{j}\right]$.

To study nonformal examples, we need to study cofibration sequences. Let $A \rightarrow X \rightarrow X / A$ be a cofibration sequence. Recall that 
$i^{*}: \mathscr{E}(X) \rightarrow \mathscr{E}(A)$ is an epimorphism [4] and let $\mathscr{E}(X, A)=\operatorname{kernel} i^{*}$. Since the composition $\mathscr{E}(X, A) \rightarrow \mathscr{E}(X) \rightarrow \mathscr{E}(A)$ is trivial, there is a factorization $\mathscr{E}(X / A) \rightarrow \mathscr{E}(X, A) \rightarrow \mathscr{E}(X)$. From the long exact homology sequence and the five lemmas, it follows that $\mathscr{E}(X / A) \rightarrow$ $\mathscr{E}(X, A)$ is a weak equivalence. Hence, we might as well use the sequence $\mathscr{E}(X, A) \rightarrow \mathscr{E}(X) \rightarrow \mathscr{E}(A)$ as the representative of $A \rightarrow X \rightarrow$ $X / A$ in the category of algebras.

Lemma 8.9. If $A \rightarrow X \rightarrow X / A$ is a cofibration sequence of finite $Q$-type nilpotent spaces with $A$ simply connected, then the sequence $\mathscr{E}(X, A) \rightarrow \mathscr{E}(X) \rightarrow \mathscr{E}(A)$ is weakly equivalent to a sequence $A_{1} \rightarrow$ $A_{2} \rightarrow A_{3}$ of connected finite type algebras with $A_{2} \rightarrow A_{3}$ surjective and $A_{1}=\operatorname{kernel}\left(A_{2} \rightarrow A_{3}\right)$.

Proof. By $C M$ 5, we can choose a surjection $D \rightarrow \mathscr{E}(A)$ where $D$ is cofibrant and acyclic. Hence, we get a map $M_{A} \otimes D \rightarrow \mathscr{E}(A)$ which is a fibration and a weak equivalence. By $C M 4$, there is a commutative diagram

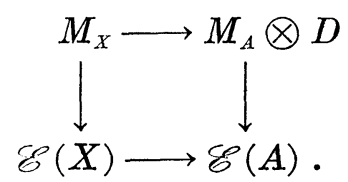

Pick a cofibrant acyclic algebra $E$ and a surjection $E \rightarrow M_{A} \otimes D$. By $C M 4$, there is a commutative diagram

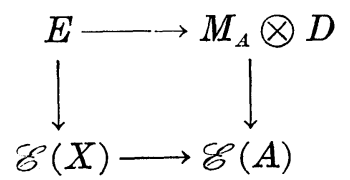

and hence

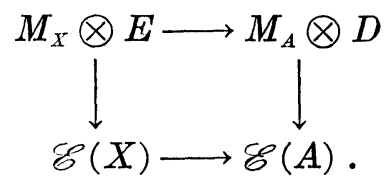

In the last commutative diagram, the vertical maps are weak equivalences and the horizontal maps are surjections. Now consider the composition $M_{X} \otimes E \rightarrow M_{A} \otimes D \rightarrow M_{A}$, where the second map is projection. If we use the same techniques that we applied to get $E$, we can find an acyclic $F$ and a surjection $F \rightarrow M_{A}$ giving a commutative diagram

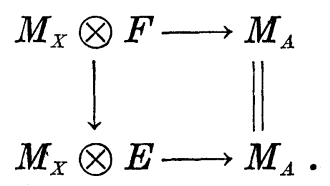

But $M_{A}$ is of finite type and $M_{A}^{1}=0$; hence, we can find an $F$ which is of finite type and connected. Set $A_{3}=M_{A}, A_{2}=M_{X} \otimes F$, and $A_{1}=\operatorname{kernel}\left(A_{2} \rightarrow A_{3}\right)$. 
Corollary 8.10. If $A \rightarrow X \rightarrow X / A$ is a cofibration sequence of finite Q-type simply connected spaces, then there is a sequence $L_{1} \rightarrow$ $L_{2} \rightarrow L_{3}$ of cofibrant Lie algebras with $L_{1}, L_{2}, L_{3}$ weakly equivalent to $L_{A}, L_{X}, L_{X / A}$, respectively, and this sequence splits into a free product when we forget the differential, that is, $L_{2}^{\#}=L_{1}^{\sharp} \vee L_{3}^{\sharp}$.

Proof. Apply the functor $\mathscr{L}$ to the sequence of coalgebras which is dual to the finite type sequence constructed in 8.9.

The next proposition is the Lie algebra version of the Hirsch lemma. It tells us how to attach a cell. Recall that $L_{S^{n}}=F[x]$, the free Lie algebra on a generator $x$ of degree $n-1$.

Proposition 8.11. Let $X$ be a finite Q-type simply connected space and let $L_{X} \rightarrow F$ be a weak equivalence with $F$ cofibrant. Let $\alpha: S^{n} \rightarrow X$ and $Y=X \bigcup_{\alpha} e^{n+1}$ with $n \geqq 1$. Then there is a weak equivalence $L_{Y} \rightarrow F^{\prime \prime}$ where $F^{\prime \prime}=F \vee F[y]$, the differential on $F^{\prime}$ extends that on $F$, and $d y$ is a chain representative for the attaching $\operatorname{map} \alpha$, degree $y=n$.

Proof. Apply 8.10 to the cofibration sequence $X \rightarrow Y \rightarrow S^{n+1}$ and let $L_{1} \rightarrow L_{2} \rightarrow L_{3}$ be the resulting sequence of Lie algebras.

Since $F$ is cofibrant, it is not hard to use $C M 4$ to get a weak equivalence $F \rightarrow L_{1}$. Consider the map $F[x]=L_{S^{n}} \rightarrow L_{X}$ and form the compositions $F[x] \rightarrow L_{X} \rightarrow F \rightarrow L_{1}$. Let $x_{2}$ and $x_{1}$ denote the images of $x$ in $F$ and $L_{1}$, respectively.

Recall the "cofibration" spectral sequence in [23, p. 262, 6.7]. $L_{2}=L_{1} \vee L_{3}$ is filtered by the degree of the $L_{3}$ factor and $E_{p, q}^{2}=$ $H_{p} L_{3} \vee H_{q} L_{1}$ with $E^{r}$ converging to $H L$.

Since $H L_{3}=F[y]$, it follows that $y$ transgresses to a generator of the kernel of $H_{n-1} L_{1} \rightarrow H_{n-1} L_{2}$. Hence we can choose a representative $y_{1}$ for $y$ in $L_{2}$ such that $d y_{1}=x_{1}$. Set $F^{\prime}=F \vee F[y]$ with $d y=x_{2}$. Map $F^{\prime} \rightarrow L_{2}$ by the map $F \rightarrow L_{1} \rightarrow L_{2}$ and $y \rightarrow y_{1}$. This map $F^{\prime} \rightarrow L_{2}$ is a weak equivalence by the comparison theorem applied to the cofibration spectral sequences of $F \rightarrow F^{\prime} \rightarrow F[y]$ and $L_{1} \rightarrow L_{2} \rightarrow L_{3}$.

Since $F^{\prime} \rightarrow L_{2}$ is a weak equivalence, the minimal model for $F^{\prime}$ is isomorphic to $L_{Y}$. That is, there is a weak equivalence $L_{Y} \rightarrow F^{\prime \prime}$.

An immediate corollary of 8.11 is the following.

Proposition 8.12. Let $X$ be a finite type $C W$ complex with a single point for its one skeleton. Let $C(X)$ denote the rational $C W$ chains on $X$. Then there is a differential on $L=F\left[s^{-1} \bar{C}(X)\right]$ such 
that there is a weak equivalence $L_{X} \rightarrow L$. In particular, $H L$ is isomorphic to $\pi(\Omega X) \otimes Q$. If $e$ is a cell of $X$, then $d s^{-1} e$ is a chain representative of the attaching map of $e$.

For example, let $X=S^{2} \vee S^{2} \bigcup_{\alpha} e^{5}$ where the attaching map $\alpha$ is the iterated Whitehead product $[[\beta, \gamma], \gamma]$ on generators $\beta, \gamma$ of $\pi_{2}\left(S^{2} \vee S^{2}\right)$. Let $F$ be the free Lie algebra on generators $x, y, z$ where degree $x=\operatorname{degree} y=1$ and degree $z=4$. Define $d x=d y=0$ and $d z=[[x, y], y]$. By 8.12, there is a weak equivalence $L_{X} \rightarrow F$. Since $F$ is a minimal Lie algebra, $L_{X}$ is isomorphic to $F$.

As the above example shows, 8.12 gives the minimal Lie algebra model when applied to a minimal $C W$ structure. Conversely, if the minimal model is known, then 8.12 gives representative cycles for the attaching maps in a minimal rational $C W$ structure. For example, 8.8 implies that, for a simply connected formal space, the attaching maps for a rational $C W$ structure can be read off from the rational homology coalgebra.

Minimal Lie algebra models can sometimes be effective in answering the following question. How many rational homotopy types are there with a fixed rational homology coalgebra $C$ ? By the remarks which follow 8.4, this is equivalent to looking at isomorphism classes of free Lie algebras $F\left[s^{-1} \bar{C}\right]$ with the quadratic term of the differential specified by $C$. Since this will be the subject of another paper, we will not go into any detail here. We leave the following straightforward exercises to the reader. There are two rational homotopy types that have the rational homology coalgebra $H\left(S^{3} \vee\right.$ $\left.S^{3} \vee S^{8} ; Q\right)$. There are countably infinite rational homotopy types that have the rational homology coalgebra $H\left(S^{3} \vee S^{3} \vee S^{12} ; Q\right)$. We were motivated to look for this second example by Stasheff.

\section{REFERENCES}

1. J. F. Adams, On the cobar construction, Proc. Nat. Acad. Sci. U.S.A., 42 (1956), 409-412.

2. A. K. Bousfield and V. K. A. M. Gugenheim, On PL deRham theory and rational homotopy type, (preprint).

3. A. K. Bousfield and D. M. Kan, Homotopy Limits, Completions and Localizations, Berlin, Heidelburg, New York: Springer-Verlag, 1972.

4. P. Deligne, P. Griffiths, J. Morgan, and D. Sullivan, Real homotopy theory of Kähler manifolds, Invent. Math., 29 (1975), 245-274.

5. S. Eilenberg and J. C. Moore, Foundations of relative homological algebra, Amer. Math. Soc. Memoirs, 55 (1965).

6. - Homology and fibrations I., Comment. Math. Helv., 40 (1966), 199-236.

7. — Limits and spectral sequences, Topology, 1 (1962), 1-23.

8. E. Friedlander, P. A. Griffiths, and J. Morgan, Homotopy theory and differential forms, Seminorio di Geometria, Firenze, 1972 (mimeographed).

9. P. Gabriel and M. Zisman, Calculus of Fractions and Homotopy Theory, Berlin, 
Heidelburg, New York: Springer-Verlag, 1966.

10. D. Husemoller, J. C. Moore, and J. Stasheff, Differential homological algebra and homogeneous spaces, J. Algebra, 5 (1974), 113-185.

11. J. M. Lemaire, Algèbres Connexes et Homologie des Espaces de Lacets, Berlin, Heidelburg, New York: Springer-Verlag, 1974.

12. M. Lazard, Sur les groupes nilpotents et les anneaux de Lie, Ann. Ec. Norm. Sup., 71 (1954), 101-190.

13. A. L. Malcev, Nilpotent groups without torsion, Izvest. Akad. Nauk SSSR, ser. Math., 13 (1949), 201-212.

14. T. Miller, Minimal Lie algebras in rational homotopy theory, University of Notre Dame dissertation, 1976.

15. T. Miller and J. Neisendorfer, Formal and coformal spaces, (preprint).

16. J. Milnor and J. C. Moore, On the structure of Hopf algebras, Ann. of Math., 81 (1965), 211-264.

17. J. C. Moore, Differential homological algebra, Actes, Congrès, Intern. Math., 1970 . Tome 1, 335-339.

18. - Some properties of the loop homology of commutative coalgebras, in the Steenrod algebra and its applications, edited by Frank Peterson. Berlin, Heidelburg, New York: Springer-Verlag, 1970.

19. What it means for a coalgebra to be simply connected, (preprint).

20. J. Neisendorfer, Rational homotopy groups of complete intersections, (preprint).

21. J. Neisendorfer and L. Taylor. Dolbeault homotopy theory, (preprint).

22. D. Quillen, Homotopical Algebra, Berlin, Heidelburg, New York: Springer-Verlag, 1967.

23. - Rational homotopy theory, Ann. of Math., 90 (1969), 205-295.

24. L. Smith, Homological algebra and the Eilenberg-Moore spectral sequence, Trans. Amer. Math. Soc., 129 (1967), 58-93.

25. - Lectures on the Eilenberg-Moore Spectral Sequence, Berlin, Heidelburg, New York: Springer-Verlag, 1970.

26. D. Sullivan, Infinitesimal computations in topology, (preprint).

27. - Topology of manifolds and differential forms, Proceedings of Conference on Manifolds, Tokyo, 1973.

28. R. G. Swan, Thom's theory of differential forms of simplicial sets, Topology, 14 (1975), 271-273.

29. H. Weyl, The Classical Groups, Princeton, 1946.

30. G. W. Whitlhead, On mappings into group-like spaces, Comment. Math., Helv. 28 (1954), 320-328.

Received October 4, 1976.

SYRACUSE UNIVERSITY

SyRaCUSE, NY 13210 


\section{PACIFIC JOURNAL OF MATHEMATICS}

\section{EDITORS}

RICHARD ARENS (Managing Editor)

University of California

Los Angeles, CA 90024

Charles W. Curtis

University of Oregon

Eugene, OR 97403

C. C. Moore

University of California

Berkeley, CA 94720

\section{J. DugundJI}

Department of Mathematics

University of Southern California

Los Angeles, CA 90007

R. FInN and J. Milgram

Stanford University

Stanford, CA 94305

\section{ASSOCIATE EDITORS}
E. F. BECKENBACH
B. H. NeumanN
F. WOLF
K. YoSHIDA

\section{SUPPORTING INSTITUTIONS}

\author{
UNIVERSITY OF BRITISH COLUMBIA \\ CALIFORNIA INSTITUTE OF TECHNOLOGY \\ UNIVERSITY OF CALIFORNIA \\ MONTANA STATE UNIVERSITY \\ UNIVERSITY OF NEVADA, RENO \\ NEW MEXICO STATE UNIVERSITY \\ OREGON STATE UNIVERSITY \\ UNIVERSITY OF OREGON
}

\author{
UNIVERSITY OF SOUTHERN CALIFORNIA \\ STANFORD UNIVERSITY \\ UNIVERSITY OF HAWAII \\ UNIVERSITY OF TOKYO \\ UNIVERSITY OF UTAH \\ WASHINGTON STATE UNIVERSITY \\ UNIVERSITY OF WASHINGTON
}

The Supporting Institutions listed above contribute to the cost of publication of this Journal, but they are not owners or publishers and have no responsibility for its content or policies.

Mathematical papers intended for publication in the Pacific Journal of Mathematics should be in typed form or offset-reproduced, (not dittoed), double spaced with large margins. Please do not use built up fractions in the text of the manuscript. However, you may use them in the displayed equations. Underline Greek letters in red, German in green, and script in blue. The first paragraph or two must be capable of being used separately as a synopsis of the entire paper. Items of the bibliography should not be cited there unless absolutely necessary, in which case they must be identified by author and journal, rather than by item number. Manuscripts, in triplicate, may be sent to any one of the editors. Please classify according to the scheme of Math. Reviews, Index to Vol. 39. All other communications should be addressed to the managing editor, or Elaine Barth, University of California, Los Angeles, California, 90024.

50 reprints to each author are provided free for each article, only if page charges have been substantially paid. Additional copies may be obtained at cost in multiples of 50 .

The Pacific Journal of Mathematics is issued monthly as of January 1966. Regular subscription rate: $\$ 72.00$ a year (6 Vols., 12 issues). Special rate: $\$ 36.00$ a year to individual members of supporting institutions.

Subscriptions, orders for numbers issued in the last three calendar years, and changes of address should be sent to Pacific Journal of Mathematics, 103 Highland Boulevard, Berkeley, California, 94708. Older back numbers obtainable from Kraus Periodicals Co., Route 100, Millwood, NY 10546.

PUBLISHED BY PACIFIC JOURNAL OF MATHEMATICS, A NON-PROFIT CORPORATION

Printed at Kokusai Bunken Insatsusha (International Academic Printing Co., Ltd.). 8-8, 3-chome, Takadanobaba, Shinjuku-ku, Tokyo 160, Japan.

Copyright (C) 1978 by Pacific Journal of Mathematics

Manufactured and first issued in Japan 


\section{Pacific Journal of Mathematics

Vol. 74, No. $2 \quad$ June, 1978

Aharon Atzmon, Spectral synthesis in some spaces of bounded continuous

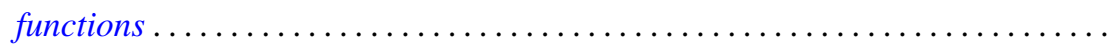

Karl Egil Aubert and Isidor Fleischer, Tensor products of ideal systems and their modules.............................................

Richard F. Basener, Several dimensional properties of the spectrum of a uniform

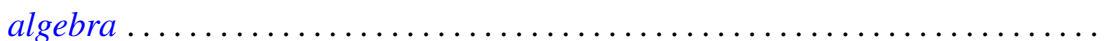

R. H. Bing and Michael Peter Starbird, Super triangulations ............. 307

Andrew Carson, Coherent polynomial rings over regular rings of finite index .....

Robert M. DeVos and Frederick W. Hartmann, Sequences of bounded summability domains .................................................

George Grätzer and R. Padmanabhan, Symmetric difference in abelian groups ....

Robert L. Griess, Jr., A remark about groups of characteristic 2-type and

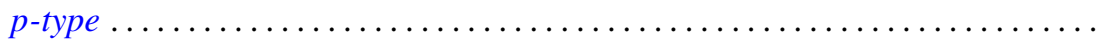

Emil Grosswald and F. J. Schnitzer, A class of modified $\zeta$ and L-functions........

Jutta Hausen and Johnny Albert Johnson, Ideals and radicals of some

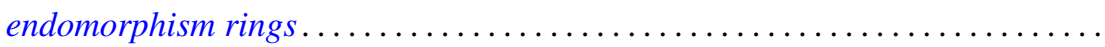

Jean Ann Larson, A solution for scattered order types of a problem of Hagendorf ............................................

Peter A. McCoy, Extremal properties of real biaxially symmetric potentials in $E^{2(\alpha+\beta+2)}$

Héctor Alfredo Merklen, Hereditary crossed product orders .

Hal G. Moore and Adil Mohamed Yaqub, Equational definability of addition in certain rings...

Robert Laurens Moore, Reductivity in $C^{*}$-algebras and essentially reductive operators. . .

Joseph Alvin Neisendorfer, Lie algebras, coalgebras and rational homotopy theory for nilpotent spaces...

William Raymond Nico, Bounded monoids

Richard Paul Osborne, Simplifying spines of 3-manifolds ...

Richard Paul Osborne, The simplest closed 3-manifolds. With an appendix by Osborne and J. Yelle.

Clayton Collier Sherman, The $K$-theory of an equicharacteristic discrete valuation ring injects into the $K$-theory of its field of quotients.... .

Mitchell Herbert Taibleson, The failure of even conjugate characterizations of $H^{1}$

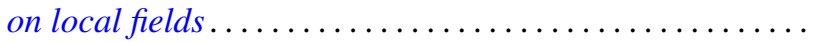

Keti Tenenblat, On characteristic hypersurfaces of submanifolds in Euclidean space ...................................

Jeffrey L. Tollefson, Involutions of Seifert fiber spaces..........

Joel Larry Weiner, An inequality involving the length, curvature, and torsions of a curve in Euclidean $n$-space .......................

Neyamat Zaheer, On generalized polars of the product of abstract homogeneous polynomials.... 